\title{
Finite-Time Universality in Nonequilibrium CFT
}

\author{
Krzysztof Gawędzki ${ }^{1}$ (D) Edwin Langmann² ED $^{2}$ \\ Per Moosavi ${ }^{2}$ (D)
}

Received: 30 November 2017 / Accepted: 6 March 2018 / Published online: 28 March 2018

(C) The Author(s) 2018

\begin{abstract}
Recently, remarkably simple exact results were presented about the dynamics of heat transport in the local Luttinger model for nonequilibrium initial states defined by position-dependent temperature profiles. We present mathematical details on how these results were obtained. We also give an alternative derivation using only algebraic relations involving the energy-momentum tensor which hold true in any unitary conformal field theory (CFT). This establishes a simple universal correspondence between initial temperature profiles and the resulting heat-wave propagation in CFT. We extend these results to larger classes of nonequilibrium states. It is proposed that such universal CFT relations provide benchmarks to identify nonuniversal properties of nonequilibrium dynamics in other models.
\end{abstract}

Keywords Nonequilibrium dynamics · Conformal field theory $\cdot$ Heat and charge transport . Luttinger model

\section{Introduction}

The study of heat, mass, charge, or spin transport in classical and quantum one-dimensional systems has a long history, see, e.g., [1-10]. Among problems that continue to make this an active field are questions concerning presence of diffusion, effects of integra-

This article is dedicated to Herbert, Jürg, and Tom.

$凶$ Per Moosavi

pmoosavi@kth.se

Krzysztof Gawędzki

kgawedzk@ens-lyon.fr

Edwin Langmann

langmann@kth.se

1 Université de Lyon, ENS de Lyon, Université Claude Bernard, CNRS, Laboratoire de Physique, 69342 Lyon, France

2 Department of Physics, KTH Royal Institute of Technology, 10691 Stockholm, Sweden 
bility, interactions, or disorder, universality, and behaviors after quantum quenches, to mention a few. Studies of such questions were further spurred by experiments on ultracold atomic gases [11,12] which recently triggered a rapid development of this field, see, e.g., [13-23]. Let us specifically mention the use of methods of conformal field theory (CFT) to gain better understanding of nonequilibrium steady states and transport in critical quantum $1 d$ systems, see [24-27] and references therein, [28] for an operatoralgebraic approach, and [29-32] that are particularly close to the context of the present paper.

In [33] two of us (E.L. and P.M.) together with Joel L. Lebowitz and Vieri Mastropietro studied in the Luttinger model the dynamics of heat transport starting from a particular class of nonequilibrium initial states. These states were given by position-dependent temperature profiles $1 / \beta(x)>0$, and the time evolution was determined by the standard translation invariant Hamiltonian

$$
H=\int_{-L / 2}^{L / 2} \mathcal{E}(x) d x,
$$

where $\mathcal{E}(x)$ is the energy density operator on a circle $S^{1}$ with circumference $L$ parameterized by the coordinate $x \in[-L / 2, L / 2] . \mathcal{E}(x)$ together with the heat current operator $\mathcal{J}(x)$ satisfy the continuity equation

$$
\partial_{t} \mathcal{E}+\partial_{x} \mathcal{J}=0
$$

with the usual Heisenberg time evolution $\mathcal{O}(t)=\mathrm{e}^{\mathrm{i} H t} \mathcal{O} \mathrm{e}^{-\mathrm{i} H t}$ for observables $\mathcal{O}=\mathcal{E}(x)$ and $\mathcal{J}(x)$. (The units are such that $\hbar=k_{B}=e=1$.) We computed the evolution of the energy density and the heat current, $\langle\mathcal{E}(x, t)\rangle_{\text {neq }}$ and $\langle\mathcal{J}(x, t)\rangle_{\text {neq }}$, using the following definition of nonequilibrium expectation values: ${ }^{1}$

$$
\langle\mathcal{O}\rangle_{\text {neq }}=\frac{\operatorname{Tr}\left(\mathrm{e}^{-\mathcal{G}} \mathcal{O}\right)}{\operatorname{Tr}\left(\mathrm{e}^{-\mathcal{G}}\right)}, \quad \mathcal{G}=\int_{-L / 2}^{L / 2} \beta(x) \mathcal{E}(x) d x .
$$

Note that the special case of constant $\beta(x)=\beta_{0}$ corresponds to the standard Gibbs equilibrium expectations

$$
\langle\mathcal{O}\rangle_{\beta_{0}}=\frac{\operatorname{Tr}\left(\mathrm{e}^{-\beta_{0} H} \mathcal{O}\right)}{\operatorname{Tr}\left(\mathrm{e}^{-\beta_{0} H}\right)}
$$

at temperature $1 / \beta_{0}$, and it is therefore natural to interpret $1 / \beta(x)$ as a position-dependent temperature profile. We stick to this interpretation throughout this paper, although a more common definition of the local temperature would link it directly to $\langle\mathcal{E}(x, t)\rangle_{\text {neq. }}$. Other interpretations of $\beta(x)$ are possible, in particular if the states in (1.3) arise as equilibria for dynamics defined by inhomogeneous Hamiltonians, as will be briefly discussed at the end of the paper. The setup considered in [33] resembled that of inhomogeneous quantum quenches [34] except that the evolution was studied after quenches from mixed states of (1.3) and it was analyzed directly in real rather than imaginary time.

To study heat transport we were particularly interested in kink-like profiles $1 / \beta(x)$ interpolating between temperatures $1 / \beta_{\mathcal{L}}$ to the far left and $1 / \beta_{\mathcal{R}}$ to the far right, see Fig. 1 . The smooth temperature profile protocol described above allows one to analytically compute the nontrivial behavior of the energy density and the heat current around the location of the kink at early times and their subsequent development into heat waves moving ballistically to the right and left. This should be contrasted with the results of the CFT description of

1 This definition differs from $\langle\mathcal{O}\rangle$ in [33] in that the thermodynamic limit is not taken in (1.3). 


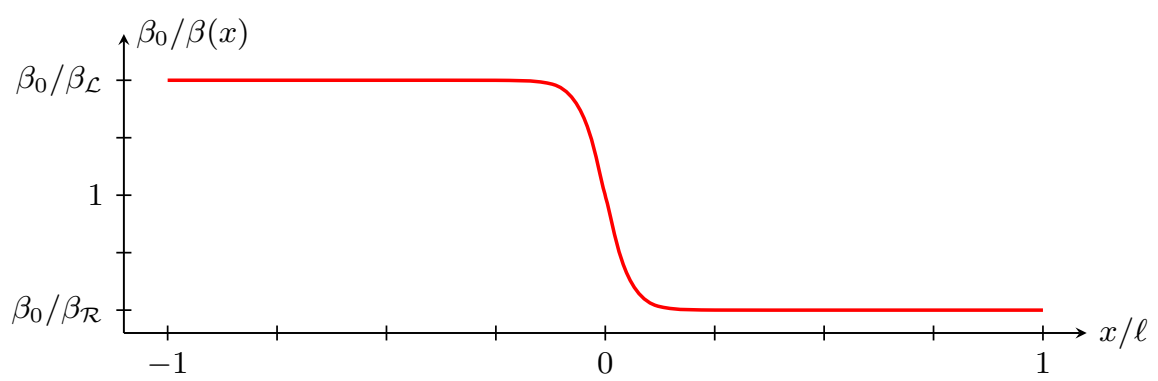

(a)

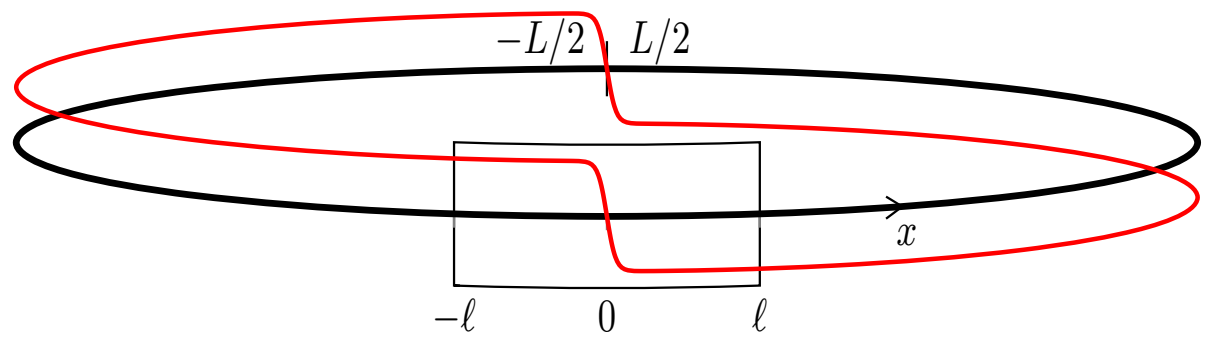

(b)

Fig. 1 Temperature profile for a the subsystem on a finite interval $[-\ell, \ell]$ with $L \gg \ell>0$ and $\mathbf{b}$ the full system with periodic boundary conditions. Note that, in addition to the kink at $x=0$, there is an opposite one at $x= \pm L / 2$, which is necessary to have a smooth periodic function. As explained in Sect. 2, the effect of this second kink is eliminated in the thermodynamic limit $L \rightarrow \infty$

the dynamics in the partitioning protocol employed in similar previous studies, see [27] and references therein. In such a description, argued to be valid after a transient time, the ballistic heat waves are compressed to simple jumps (shocks) without internal structure moving away from the contact point. In the smooth initial states that we consider, this happens only in the limit when $t$ and $x$ are sent to infinity at the same rate, as such a limit wipes out the nontrivial internal structure of the heat waves. The evolution of the energy density and the heat current obtained in [33] permits then to better understand the shortcomings of the partitioning protocol. It also sheds a new interesting light on transport in integrable systems and, in particular, on how its universal features [35] emerge at long times for a large class of nonequilibrium initial states, see [36] and also Sect. 4.3 below for a related discussion of charge transport in the Luttinger model. As a representative example, we plot $\langle\mathcal{E}(x, t)\rangle_{\text {neq }}$ and $\langle\mathcal{J}(x, t)\rangle_{\text {neq }}$ in Fig. 2 at four times for the Luttinger model with local interactions (defined in more detail below) starting from the kink-like temperature profile in Fig. 1. Note a peak and a dip in the energy density at time $t=0$ in the region where the temperature changes and how this local shape evolves into heat waves. This is accompanied by a universal heat current building up in the region between the two heat-wave fronts. We note that, for local interactions, the wave fronts preserve their shapes in time. For the Luttinger model with nonlocal interactions, there are additional dispersive effects, which, however, eventually become unobservable in any finite region as the wave fronts leave such regions in finite time, see Fig. 1 in [33]. In the remainder of this paper we restrict our discussion to the local case. 

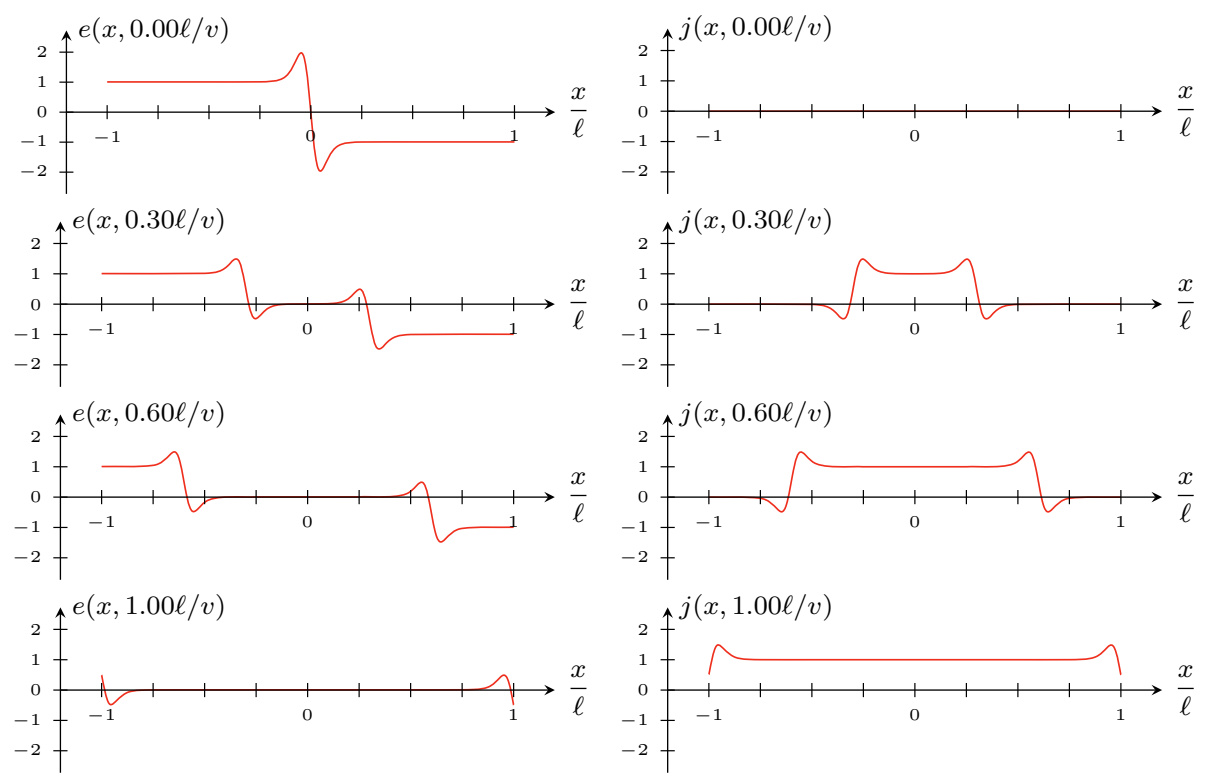

Fig. 2 Plots of the energy density $e(x, t)=v\left[\langle\mathcal{E}(x, t)\rangle_{\text {neq }}^{\infty}-\langle\mathcal{E}(x, t)\rangle_{\bar{\beta}}^{\infty}\right] / J$ and the heat current $j(x, t)=\langle\mathcal{J}(x, t)\rangle_{\text {neq }}^{\infty} / J$ for the Luttinger model in a finite interval $[-\ell, \ell]$ around $x=0$ rescaled by $J=\lim _{t \rightarrow \infty}\langle\mathcal{J}(x, t)\rangle_{\text {neq }}^{\infty}=(\pi / 12)\left(\beta_{\mathcal{L}}^{-2}-\beta_{\mathcal{R}}^{-2}\right)$ for different times in the nonequilibrium state with the inverse-temperature profile given by (2.1) and (2.3). The parameters are $\beta_{\mathcal{L}}=19.9, \beta_{\mathcal{R}}=20.1, \delta / \ell=0.06$, and $v / \ell=0.025$

In the local Luttinger model, the energy density is given formally by ${ }^{2}$

$\mathcal{E}(x)=\sum_{r= \pm} \frac{v_{F}}{2}:\left[\psi_{r}^{\dagger}(x)\left(-\mathrm{i} r \partial_{x}\right) \psi_{r}(x)+\right.$ H.c. $]:+\lambda \sum_{r, r^{\prime}= \pm}: \psi_{r}^{\dagger}(x) \psi_{r}(x):: \psi_{r^{\prime}}^{\dagger}(x) \psi_{r^{\prime}}(x):-\mathcal{E}_{0}$

with fermionic field operators $\psi_{ \pm}(x)$ (with antiperiodic boundary conditions) obeying the usual canonical anticommutation relations $\left\{\psi_{r}(x), \psi_{r^{\prime}}^{\dagger}(y)\right\}=\delta_{r, r^{\prime}} \delta(x-y)$, etc., $: \cdots:$ denoting Wick (normal) ordering, the bare Fermi velocity $v_{F}>0$, and the coupling strength $\lambda>-\pi v_{F} / 2$. The (diverging) constant $\mathcal{E}_{0}$ subtracts the ground-state energy density up to the finite contribution $-\pi v /\left(6 L^{2}\right)$ left for later convenience. The heat current is given by $\mathcal{J}(x)=v^{2} \mathcal{P}(x)$ with the momentum density operator

$$
\mathcal{P}(x)=\sum_{r= \pm} \frac{1}{2}:\left[\psi_{r}^{\dagger}(x)\left(-\mathrm{i} \partial_{x}\right) \psi_{r}(x)+\text { H.c. }\right]:
$$

and the plasmon velocity $v=v_{F} \sqrt{1+2 \lambda /\left(\pi v_{F}\right)}$ [36,39]. We found in [33] the following exact expectation values of the energy density and the heat current in the thermodynamic limit marked by the superscript ${ }^{\infty}$ on the expectations: ${ }^{3}$

$$
\left\langle\left.\mathcal{E}(x, t)\right|_{\text {neq }} ^{\infty}=\frac{1}{2}\left[F\left(x^{-}\right)+F\left(x^{+}\right)\right], \quad\left\langle\left.\mathcal{J}(x, t)\right|_{\text {neq }} ^{\infty}=\frac{v}{2}\left[F\left(x^{-}\right)-F\left(x^{+}\right)\right],\right.\right.
$$

\footnotetext{
2 One can make this mathematically precise by considering the analogous expression for a nonlocal interaction and then taking the local limit [37] in an appropriate bosonized Fock space, see, e.g., [38].

3 The symbols $\mathcal{E}, F$, and $f$ here correspond to $\mathcal{H}, G$, and $g$ in [33].
} 
where $x^{ \pm}=x \pm v t$ are the light-cone coordinates and the function

$$
F(x)=\frac{\pi}{6 v} \frac{1}{\beta(x)^{2}}+\frac{v}{12 \pi}\left(\frac{\beta^{\prime \prime}(x)}{\beta(x)}-\frac{1}{2}\left(\frac{\beta^{\prime}(x)}{\beta(x)}\right)^{2}\right)
$$

is determined by the temperature profile. We also observed that $F(x)$ can be written in terms of the Schwarzian derivative

$$
(S f)(x)=\frac{f^{\prime \prime \prime}(x)}{f^{\prime}(x)}-\frac{3}{2}\left(\frac{f^{\prime \prime}(x)}{f^{\prime}(x)}\right)^{2}
$$

of the function

$$
f(x)=\int_{0}^{x} \frac{\beta_{0}}{\beta\left(x^{\prime}\right)} d x^{\prime}
$$

as

$$
F(x)=\frac{\pi}{6 v \beta_{0}^{2}} f^{\prime}(x)^{2}-\frac{v}{12 \pi}(S f)(x)
$$

for some constant $\beta_{0}>0$. Noting that Schwarzian derivatives appear in CFT [40] and that the local Luttinger model is a CFT, we also argued that it should be possible to obtain the result in (1.7) and (1.8) in a simpler way using conformal transformations, and that it may be possible to also obtain expectation values of other observables in that way [33]. Our main result in this paper is to show that this is indeed the case.

The method used in [33] was perturbative in a small parameter $\epsilon$ measuring the distance to equilibrium in the initial state (i.e., the case $\epsilon=0$ corresponds to the Gibbs state). This method is general, but generically one can only obtain useful low-order results. In the special case of local interactions, however, we were able to push the computations to all orders in $\epsilon$, and, summing the resulting infinite series, we obtained the results in (1.7) and (1.8). In this paper we give a simpler derivation of these results extending them to all unitary CFT models, including models at finite $L$, and to other observables.

Our analysis is based on the Minkowskian version of CFT, and it generalizes to other classes of nonequilibrium states. One such generalization is (1.3) but where, in addition to the temperature profile $1 / \beta(x)$, we also allow for a "velocity" profile $v(x)$ taking

$$
\mathcal{G}=\int_{-L / 2}^{L / 2} \beta(x)[\mathcal{E}(x)+v(x) \mathcal{P}(x)] d x
$$

for $|v(x)|<v$ (this condition ensures that $\mathcal{G}$ is positive). Note that for constant profiles this is a generalization [41] of the Gibbs state in (1.4) where $\beta_{0} H$ is replaced by $\beta_{0}\left(H+v_{0} P\right)$ with the momentum operator

$$
P=\int_{-L / 2}^{L / 2} \mathcal{P}(x) d x .
$$

This may be also be viewed in CFT as a Lorentz boost of the ordinary Gibbs state [27]. As will be shown, the corresponding generalization of the result in (1.7) is obtained by replacing $F\left(x^{\mp}\right)$ by $F_{ \pm}\left(x^{\mp}\right)$ given by (1.8) with the right-hand side multiplied by the central charge $c$ of CFT (which is equal to 1 for the local Luttinger model) and with $\beta(x)$ replaced by

$$
\beta_{ \pm}(x)=\beta(x)[1 \pm v(x) / v] .
$$

In particular, in the long-time limit

$$
\lim _{t \rightarrow \infty}\langle\mathcal{E}(x, t)\rangle_{\text {neq }}^{\infty}=\frac{\pi c}{12 v}\left(\beta_{+, \mathcal{L}}^{-2}+\beta_{-, \mathcal{R}}^{-2}\right), \quad \lim _{t \rightarrow \infty}\langle\mathcal{J}(x, t)\rangle_{\text {neq }}^{\infty}=\frac{\pi c}{12}\left(\beta_{+, \mathcal{L}}^{-2}-\beta_{-, \mathcal{R}}^{-2}\right),
$$


where $\beta_{ \pm, \mathcal{L}}$ and $\beta_{ \pm, \mathcal{R}}$ are the asymptotic values of $\beta_{ \pm}(x)$ to the left and to the right, respectively. $^{4}$

Finally, for CFTs with a double U(1) current algebra (e.g., the Luttinger model itself), we may also handle chemical-potential profiles $\mu_{ \pm}(x)$ in addition to temperature profiles $1 / \beta_{ \pm}(x)$, possibly different for right and left movers. In this case, the functions $F_{ \pm}(x)$ pick up an additional term proportional to $\mu_{ \pm}(x)^{2}$. This is the largest class of nonequilibrium states that we consider. They are defined as in (1.3) but with $\mathcal{G}$ in (4.40), see Sect. 4.3 for details.

The above results show that in CFT there is a universal relation between the initial temperature, velocity, and chemical-potential profiles and the resulting heat- and density-wave propagation even at finite times. ${ }^{5}$ We believe that this provides a useful benchmark for other models as follows. Typically, finite-time results are model-dependent, and more universal behavior is only obtained at long times [27]. As an example, we mention the nonlocal Luttinger model which exhibits finite-time dispersion effects depending on short-distance details of the interaction potential [33]. However, these effects have some qualitative features that are always present. We postulate that the CFT results have to be subtracted in order to identify the effects that come from the microscopic details in the propagation of the heat or density waves emanating from the inhomogeneities of the initial state. In addition, such a subtraction should allow to identify the time scales when such model-dependent effects are important and when not. Moreover, it was argued in [19] that integrable systems come in two kinds: those that are purely ballistic and those with a nonzero diffusive contribution. For heat transport, in particular, one way to view this is through the thermal conductivity in the frequency domain

$$
\operatorname{Re} \kappa_{\text {th }}(\omega)=\pi D_{\text {th }} \delta(\omega)+\operatorname{Re} \kappa_{\text {th }}^{\text {reg }}(\omega),
$$

where a nonzero thermal Drude weight $D_{\text {th }}$ indicates the presence of a ballistic contribution and a finite nonzero value of the regular part $\operatorname{Re} \kappa_{\text {th }}^{\mathrm{reg}}(\omega)$ at $\omega=0$ signals the presence of a diffusive component in the heat transport [9]. It is known that "pure" CFT captures the ballistic part [27], with $\operatorname{Re} \kappa_{\text {th }}^{\text {reg }}=0$. This also follows from our results. The cause of dispersive and diffusive effects thus must come from short-distance details, randomness, or other relevant perturbations, see, e.g., [42] for a recent discussion of that issue within CFT.

The plan of the rest of this paper is as follows. In Sect. 2, we sketch the original derivation of the result in (1.7) and (1.8) and explain the physical significance of the limit $L \rightarrow \infty$ since this is also relevant for our more direct CFT argument. We also present special integrals whose exact evaluation was the key to this result and which, as we believe, are interesting in their own right. The reader may skip the second half of Sect. 2 without loss of continuity. The CFT derivation is given in Sect. 3. After collecting the results about Minkowski-space CFT that are needed in Sect. 3.1, we show in Sect. 3.2 how to use conformal transformations to straighten out position-dependent temperature profiles $1 / \beta(x)$ on a periodic interval. This allows one to exactly map the nonequilibrium expectations in CFT to the corresponding equilibrium ones. We make this mapping explicit for products of the components of the energy-momentum tensor. In Sect. 3.3, we study the thermodynamic limit of the finite-volume relations which allows one to treat temperature profiles on the infinite line with different asymptotic values on the left and right sides. As a byproduct, we calculate the thermal Drude weight. Section 4 is devoted to various generalizations. In Sect. 4.1, we briefly discuss the correlators of primary fields. In Sect. 4.2, we consider states with different temperature profiles for the right and

\footnotetext{
4 To avoid confusion, we stress that our subscripts \pm refer to right $(+)$ and left $(-)$ movers.

5 Here we use the term "universal" as referring to the same form in different CFTs rather than to the independence of the microscopic details of the models.
} 
left movers. They form a class of nonequilibrium states preserved by the Schrödinger-picture dynamics that lead to simple examples of generalized Gibbs states at long times. In Sect. 4.3, we extend the analysis to CFTs with a U(1) current algebra and states with temperature and chemical-potential profiles. For the Luttinger model we discuss how this implies universality of conductance for both the charge and axial currents, generalizing previous results in [36], see also [43]. Finally, in Sect. 5, we make contact with [29-32], discussing the dynamics preserving states in (1.3) and the related Euclidian CFT description. We end with conclusions and directions for further developments in Sect. 6. The Appendix contains some mathematical details on the special integrals mentioned above.

\section{Perturbative Derivation and Remarkable Integrals}

The perturbative computation method used in [33] is based on introducing an expansion parameter $\epsilon$ measuring the deviation of the temperature profile from constant temperature $1 / \bar{\beta}$ as follows:

$$
\beta(x)=\bar{\beta}[1+\epsilon W(x)]
$$

with $W(x)$ a function defined by this relation.

In [33] we were mainly interested in kink-like functions where $W(x)$ becomes (say) $1 / 2$ and $-1 / 2$ to the far left and right, respectively, so that $\bar{\beta}=\left(\beta_{\mathcal{L}}+\beta_{\mathcal{R}}\right) / 2$ and $\epsilon=\left(\beta_{\mathcal{L}}-\beta_{\mathcal{R}}\right) / \bar{\beta}$ in terms of the asymptotic values. On the other hand, for technical reasons, we used a model on a circle with circumference $L<\infty$, which at first sight seems incompatible: a smooth function $W(x)$ on the circle with a single kink is not possible, and there has to be at least one other opposite one. As an example, consider the periodic function

$$
W(x)=-\frac{1}{2} \tanh \left(\frac{L}{2 \pi \delta} \sin \left(\frac{2 \pi x}{L}\right)\right) \quad(\delta>0),
$$

which is kink-like in the vicinity of $x=0$ as desired but has an opposite kink in the vicinity of $x= \pm L / 2$ and leads (for negative $\epsilon$ ) to the temperature profile of Fig. 1. The effect of the additional step around $\pm L / 2$ can be eliminated by computing results $\langle\mathcal{O}(t)\rangle_{\text {neq }}$ for $L<\infty$ at finite times $t$, and then taking the limit $L \rightarrow \infty$ [33] in which (2.2) turns into

$$
W(x)=-\frac{1}{2} \tanh \left(\frac{x}{\delta}\right) .
$$

In this way technical problems with $L=\infty$ on the level of quantum field theory are avoided and, at the same time, boundary conditions have no influence on the final results. The physical interpretation is that the kink at $x= \pm L / 2$ is "behind the moon" and does not affect the physics in any finite region at times significantly smaller than $L / v$, which is a time scale that becomes infinite in the limit $L \rightarrow \infty$.

In the rest of this section we sketch the perturbative derivation of the result in (1.7) and (1.8), concentrating on remarkable integrals which were the key to this results. The readers mainly interested in our CFT derivation may pass directly to Sect. 3 without loss of continuity.

With the inverse-temperature profile (2.1), one can use the Dyson series to obtain an expansion

$$
\langle\mathcal{O}(x, t)\rangle_{\text {neq }}=\langle\mathcal{O}(x, t)\rangle_{\bar{\beta}}+\epsilon\langle\mathcal{O}(x)\rangle_{1}+\epsilon^{2}\langle\mathcal{O}(x, t)\rangle_{2}+\cdots
$$

for any local observable $\mathcal{O}(x, t)$, such as $\mathcal{E}(x, t)$ and $\mathcal{J}(x, t)$. The leading term in (2.4) is the equilibrium expectation value (which is time independent, i.e., $\left.\langle\mathcal{O}(x, t)\rangle_{\bar{\beta}}=\langle\mathcal{O}(x)\rangle_{\bar{\beta}}\right)$, and the $n$-th order term is an $(n+1)$-point correlation function for $n=1,2, \ldots$ [33]. This 
method works, in principle, for any model but, in practice, it is difficult to go beyond leading order $n=1$. For a quasi-free bosonic model, to which the Luttinger model reduces, one can use general mathematical results [44-46] to replace the many-body computation by a much simpler one-particle one, and this makes it possible to extend the calculation to all orders [33].

In particular, for the local Luttinger model, after taking the limit $L \rightarrow \infty$, this method gives (1.7) with

$$
F(x)=\frac{\pi}{6 v \bar{\beta}^{2}}+\sum_{n=1}^{\infty} \epsilon^{n} F_{n}(x),
$$

where

$$
\begin{aligned}
F_{n}(x) & =\frac{v}{4 \pi} \int_{\mathbb{R}^{n}} I_{n}\left(q_{1}, \ldots, q_{n}\right)\left(\prod_{j=1}^{n} \hat{W}\left(q_{j}\right) \mathrm{e}^{\mathrm{i} q_{j} x}\right) \frac{d^{n} q}{(2 \pi)^{n}}, \\
I_{n}\left(q_{1}, \ldots, q_{n}\right) & =\frac{2}{v \bar{\beta}} \int_{\mathbb{R}} \sum_{v \in(2 \pi / \bar{\beta}) \mathbb{Z}}\left(\prod_{j=0}^{n} \frac{v\left(p+Q_{j}\right)}{\mathrm{i} v-v\left(p+Q_{j}\right)}\right) d p, \\
\hat{W}(q) & =\int_{\mathbb{R}} W(x) \mathrm{e}^{-\mathrm{i} q x} d x, \quad Q_{j}=\sum_{k=j+1}^{n} q_{k} .
\end{aligned}
$$

It is interesting to note that the limit $L \rightarrow \infty$ is not only useful to eliminate the effect of boundary conditions (as explained) but also computationally: this limit turns Riemann sums into integrals and eliminates zero-mode contributions which would be more difficult to handle [33].

The integrals in (2.7) are certainly nontrivial, but we found that they all can be computed exactly, giving the result in Lemma 2.1, and this was the key that led to (1.8).

Lemma 2.1 For all $n=1,2, \ldots$,

$$
\begin{aligned}
& I_{n}\left(q_{1}, \ldots, q_{n}\right) \\
& \quad=(-1)^{n}\left\{\frac{n+1}{6}\left(\frac{2 \pi}{v \bar{\beta}}\right)^{2}+\frac{2}{(n+1)(n+2)} \sum_{j=0}^{n} Q_{j}^{2}-\frac{4}{n(n+1)(n+2)} \sum_{0 \leq j<k \leq n} Q_{j} Q_{k}\right\}
\end{aligned}
$$

with $Q_{j}$ defined in (2.8).

A proof can be found in the Appendix.

It is remarkable that the result are even second order polynomials in the variables $q_{j}$. It is clear from (2.6) that the constant term leads to contributions to $F_{n}(x)$ which are proportional to $W(x)^{n}$, whereas the terms with $q_{j}^{2}$ and $q_{j} q_{k}, j \neq k$, lead to $W(x)^{n-2} W^{\prime \prime}(x)$ and $W(x)^{n-2} W^{\prime}(x)^{2}$, respectively. Thus, the special form of the integrals in Lemma 2.1 implies that we get at most terms involving second derivatives of $W(x)$. The explicit expression of these integrals allows one to compute $F_{n}(x)$ exactly, and the result is simple enough to analytically sum the series in (2.5), which gives the result in (1.8) [33].

We describe this computation above since it allows one to interpret the argument in the next section as a partial proof of Lemma 2.1. Such a proof is only partial since all terms with $q_{j}^{2}$ are identified with (say) $q_{1}^{2}$, and all terms with $q_{j} q_{k}$ for $j \neq k$ are identified with $q_{1} q_{2}$. This identification is also useful in order to explicitly derive (1.8) from Lemma 2.1, see Eq. 
(A4) in [33], which is implied by (2.9). Thus, the exact integrals in Lemma 2.1 contain more information than the result in (1.8). Since nontrivial integrals that can be computed exactly are rare and often not only have one but several applications in physics, we prove (2.9) in this paper. Moreover, since our derivation of (1.7) and (1.8) in the next section works even for finite $L$, it suggests interesting Riemann sum generalizations of the exact integrals in Lemma 2.1. We believe it would be interesting to work them out, but this is left for a future study.

We finally mention that our argument in the next section allows one to interpret the computation described above as a derivation of the conformal anomaly in CFT by a direct computation, see (3.7).

\section{CFT Derivation}

\subsection{CFT in Minkowski Space}

We consider the Minkowskian version of a unitary two-dimensional CFT where space is a circle $S^{1}$ parameterized by the periodic coordinate $x$ with the basic range $-L / 2 \leq x \leq L / 2$ and where $t \in \mathbb{R}$ is time. We keep the propagation speed $v$ in our equations to clearly indicate how it effects the (otherwise) universal law relating the temperature profiles to the heat-wave dynamics.

The basic objects of such a CFT are the periodic light-cone components $T_{ \pm}\left(x^{\mp}\right)=$ $T_{ \pm}\left(x^{\mp}+L\right)$ of the energy-momentum tensor ${ }^{6}$ where, as before, $x^{ \pm}=x \pm v t$. They are distributions with values in the self-adjoint operators on the Hilbert space of states of the theory that satisfy the equal-time commutation relations

$$
\begin{aligned}
& {\left[T_{ \pm}(x), T_{ \pm}(y)\right]=\mp 2 \mathrm{i} \delta^{\prime}(x-y) T_{ \pm}(y) \pm \mathrm{i} \delta(x-y) T_{ \pm}^{\prime}(y) \pm \frac{c}{24 \pi} \mathrm{i} \delta^{\prime \prime \prime}(x-y),} \\
& {\left[T_{ \pm}(x), T_{\mp}(y)\right]=0,}
\end{aligned}
$$

where $\delta(x)$ stands for the $L$-periodized $\delta$-function. The real number $c$ is the central charge of the theory. In terms of the Fourier modes,

$$
T_{ \pm}(x)=\frac{2 \pi}{L^{2}} \sum_{n=-\infty}^{\infty} \mathrm{e}^{ \pm \frac{2 \pi \mathrm{i} n x}{L}}\left(L_{n}^{ \pm}-\frac{c}{24} \delta_{n, 0}\right),
$$

the commutation relations in (3.1) and (3.2) reduce to those of the Virasoro algebra,

$$
\left[L_{n}^{ \pm}, L_{m}^{ \pm}\right]=(n-m) L_{n+m}^{ \pm}+\frac{c}{12}\left(n^{3}-n\right) \delta_{n+m, 0}, \quad\left[L_{n}^{ \pm}, L_{m}^{\mp}\right]=0 .
$$

Technically, we assume that the Hilbert space of the theory is a (possibly infinite) direct sum of unitary highest-weight representations of two commuting copies of the Virasoro algebra. The local Luttinger model is an example with $c=1$ of such a theory where

$$
T_{ \pm}(x)=\pi: \tilde{\rho}_{ \pm}(x)^{2}:-\frac{\pi}{12 L^{2}}, \quad \tilde{\rho}_{ \pm}(x)=\rho_{ \pm}(x) \cosh \varphi-\rho_{\mp}(x) \sinh \varphi
$$

\footnotetext{
6 In the more standard notation for the energy-momentum tensor components in light-cone coordinates, $T_{+}=T_{--}, T_{-}=T_{++}$and $T_{+-}=0=T_{-+}$.
} 
with the fermion densities $\rho_{ \pm}(x)=: \psi_{ \pm}^{\dagger}(x) \psi_{ \pm}(x)$ : and tanh $2 \varphi=-\lambda /\left(\pi v_{F}+\lambda\right)$. A related quantity describing the interactions is the Luttinger parameter ${ }^{7} K=\mathrm{e}^{2 \varphi}$. The effective densities $\tilde{\rho}_{ \pm}$act in a direct sum of bosonic Fock spaces that contains the interacting vacuum $|\Psi\rangle$ and the Wick ordering in (3.5) is with respect to $|\Psi\rangle[37,38]$. In the following arguments, the explicit form of the operators $T_{ \pm}(x)$ is not used.

Let $\widetilde{\operatorname{Diff}}_{+}\left(S^{1}\right)$ denote the covering group of the group $\operatorname{Diff}_{+}\left(S^{1}\right)$ of orientation-preserving diffeomorphisms of the circle. The elements of $\widetilde{\mathrm{Diff}_{+}}\left(S^{1}\right)$ are represented by smooth functions $x \mapsto f(x)$ on $\mathbb{R}$ such that $f(x+L)=f(x)+L$ and $f^{\prime}(x)>0$, with functions $f(x)$ and $f(x)+n L$ corresponding to the same diffeomorphism in $\operatorname{Diff}_{+}\left(S^{1}\right)$. The operatorvalued distributions $T_{ \pm}$generate two commuting projective unitary representations $U_{ \pm}$of $\widetilde{\operatorname{Diff}}_{+}\left(S^{1}\right)$ on the Hilbert space of the theory such that for infinitesimal diffeomorphisms $f(x)=x+\varepsilon \zeta(x)$ one has

$$
U_{ \pm}(f)=I \mp \mathrm{i} \varepsilon \int_{-L / 2}^{L / 2} \zeta(x) T_{ \pm}(x) d x+o(\varepsilon)
$$

and under the adjoint action

$$
U_{ \pm}(f) T_{ \pm}(x) U_{ \pm}(f)^{-1}=f^{\prime}(x)^{2} T_{ \pm}(f(x))-\frac{c}{24 \pi}(S f)(x)
$$

with the Schwarzian derivative $(S f)(x)$ given by (1.9). This was proven in [47] for the unitary highest-weight representations of the Virasoro algebra and carries over to the present context. The adjoint action of $U_{ \pm}(f)$ preserves $T_{\mp}$.

The energy-momentum tensor determines the Hamiltonian,

$$
H=v \int_{-L / 2}^{L / 2}\left[T_{+}(x)+T_{-}(x)\right] d x,
$$

and under the Heisenberg picture evolution, $T_{ \pm}(x, t)=T_{ \pm}\left(x^{\mp}\right)$ as claimed above. The energy and momentum density operators

$$
\mathcal{E}(x, t)=v\left[T_{+}\left(x^{-}\right)+T_{-}\left(x^{+}\right)\right], \quad \mathcal{P}(x, t)=T_{+}\left(x^{-}\right)-T_{-}\left(x^{+}\right)
$$

satisfy the continuity equations

$$
\partial_{t} \mathcal{E}+v^{2} \partial_{x} \mathcal{P}=0, \quad \partial_{t} \mathcal{P}+\partial_{x} \mathcal{E}=0 .
$$

As we shall see, the finite-volume nonequilibrium expectation values in (1.3) for $\mathcal{O}=\mathcal{E}(x)$ and $\mathcal{J}(x)=v^{2} \mathcal{P}(x)$ are well defined for any such CFT provided the inverse-temperature profile is periodic.

\subsection{Relating Nonequilibrium to Equilibrium Expectations}

From the above, it is clear that the calculation of the time evolution of the nonequilibrium expectation values of the energy density and current operators is equivalent to computing $\left\langle T_{ \pm}\left(x^{\mp}\right)\right\rangle_{\text {neq }}$.

Let us denote $U(f)=U_{+}(f) U_{-}(f)$ for $f \in \widetilde{\operatorname{Diff}}_{+}\left(S^{1}\right)$. The key observation is that it is possible to find an $f$ such that

$$
U(f) \mathcal{G} U(f)^{-1}=\beta_{0} H+\text { const }
$$

7 Equation (3.5) holds also for the local Luttinger model with two coupling constants $g_{2}$ and $g_{4}$ [39] with $K=\mathrm{e}^{2 \varphi}$ dependent on $g_{2}$ and $g_{4}$. 
for some constant $\beta_{0}>0$. In order to see this, take the function $f$ given by (1.10) with the constant $\beta_{0}$ determined by

$$
\frac{1}{\beta_{0}}=\frac{1}{L} \int_{-L / 2}^{L / 2} \frac{1}{\beta\left(x^{\prime}\right)} d x^{\prime} .
$$

The above choice of $\beta_{0}$ ensures that $f(x+L)=f(x)+L$ and thus that $f$ defines an element in $\widetilde{\operatorname{Diff}}_{+}\left(S^{1}\right)$. Using this function $f$, it follows from (1.3), (3.7), and (3.9) that

$$
\begin{gathered}
U(f) \mathcal{G} U(f)^{-1}=v \int_{-L / 2}^{L / 2} \beta(x)\left[U_{+}(f) T_{+}(x) U_{+}(f)^{-1}+U_{-}(f) T_{-}(x) U_{-}(f)^{-1}\right] d x \\
=v \int_{-L / 2}^{L / 2} \beta(x) f^{\prime}(x)^{2}\left[T_{+}(f(x))+T_{-}(f(x))\right] d x-\frac{c v}{12 \pi} \int_{-L / 2}^{L / 2} \beta(x)(S f)(x) d x .
\end{gathered}
$$

Upon using the relation $f^{\prime}(x)=\beta_{0} / \beta(x)$ for the derivative of $f$, this reduces to

$$
\begin{aligned}
U(f) \mathcal{G} U(f)^{-1}= & v \beta_{0} \int_{-L / 2}^{L / 2} f^{\prime}(x)\left[T_{+}(f(x))+T_{-}(f(x))\right] d x \\
& -\frac{c v}{12 \pi} \int_{-L / 2}^{L / 2} \beta(x)(S f)(x) d x \\
= & v \beta_{0} \int_{-L / 2}^{L / 2}\left[T_{+}(y)+T_{-}(y)\right] d y-\frac{c v}{12 \pi} \int_{-L / 2}^{L / 2} \beta(x)(S f)(x) d x,
\end{aligned}
$$

where the last equality follows by the change of variables $y=f(x)$. This establishes (3.11). In short, the conjugation with $U(f)$ straightens out the temperature profile.

From the definition in (1.3) and the relation in (3.11), we infer that

$$
\langle\mathcal{O}\rangle_{\text {neq }}=\frac{\operatorname{Tr}\left(\mathrm{e}^{-U(f) \mathcal{G} U(f)^{-1}} U(f) \mathcal{O} U(f)^{-1}\right)}{\operatorname{Tr}\left(\mathrm{e}^{\left.-U(f) \mathcal{G} U(f)^{-1}\right)}\right.}=\left\langle U(f) \mathcal{O} U(f)^{-1}\right\rangle_{\beta_{0}} .
$$

This translates the nonequilibrium expectations to the equilibrium ones defined by (1.4). For $x^{-r}=x-r v t$ and

$$
\mathcal{O}=\prod_{j} T_{r_{j}}\left(x_{j}^{-r_{j}}\right)
$$

(with noncoincident points), using again (3.7), we obtain the relation

$$
\left\langle\prod_{j} T_{r_{j}}\left(x_{j}^{-r_{j}}\right)\right\rangle_{\text {neq }}=\left\langle\prod_{j}\left(f^{\prime}\left(x_{j}^{-r_{j}}\right)^{2} T_{r_{j}}\left(f\left(x_{j}^{-r_{j}}\right)\right)-\frac{c}{24 \pi}(S f)\left(x_{j}^{-r_{j}}\right)\right)\right\rangle_{\beta_{0}}
$$

for $f$ given by (1.10) and (3.12). In particular,

$$
\left\langle T_{ \pm}\left(x^{\mp}\right)\right\rangle_{\text {neq }}=f^{\prime}\left(x^{\mp}\right)^{2}\left\langle T_{ \pm}\left(f\left(x^{\mp}\right)\right)\right\rangle_{\beta_{0}}-\frac{c}{24 \pi}(S f)\left(x^{\mp}\right) .
$$

The Gibbs state is translation invariant so that the expectations $\left\langle\left. T_{ \pm}(y)\right|_{\beta_{0}}\right.$ do not depend on $y$, but they depend, in general, on $v \beta_{0}$ and $L$. By scaling, however, $\left(v \beta_{0}\right)^{2}\left\langle\left. T_{ \pm}(y)\right|_{\beta_{0}}\right.$ depends only on $v \beta_{0} / L$ but in a way dependent on the representation content of the CFT. As we shall see, what is universal, depending only on the central charge, is the $L \rightarrow \infty$ limit of $\left(v \beta_{0}\right)^{2}\left\langle T_{ \pm}(y)\right\rangle_{\beta_{0}}$. 


\subsection{Thermodynamic Limit}

Let us consider the limit $L \rightarrow \infty$ of the nonequilibrium expectations in (3.17). For a large class of kink-like $\beta(x)$ profiles (not necessarily symmetric) with an antikink around $\pm L / 2$,

$$
\beta_{0}^{-1}=\frac{1}{2}\left(\beta_{\mathcal{L}}^{-1}+\beta_{\mathcal{R}}^{-1}\right)+O\left(L^{-1}\right),
$$

where $\beta_{\mathcal{L}}$ and $\beta_{\mathcal{R}}$ are the asymptotic values of the plateau to the left and to the right of the kink. ${ }^{8}$ Similarly, for fixed $x, \beta(x)$ will stabilize up to $O\left(L^{-1}\right)$ terms with any trace of the antikink gradually wiped out, and so does the function $f$ given by (1.10). The question about the large- $L$ limit of the nonequilibrium expectations in (3.17) then boils down to the one for the equilibrium expectations $\left\langle\prod_{j} T_{r_{j}}\left(x_{j}\right)\right\rangle_{\beta_{0}}$, where, by rescaling, $\beta_{0}$ may be set to its asymptotic value and the insertion points are allowed to have $O\left(L^{-1}\right)$ variations.

In CFT the control of the thermodynamic limit of the equilibrium expectations is an easy exercise. The thermal expectations like those mentioned above may be viewed as the ones in the Euclidean theory on the torus $S^{1} \times S^{1}$ where the circles have circumferences $L$ and $v \beta_{0}$, respectively. In a modular invariant CFT [40], they also have a dual representation

$$
\left\langle\prod_{j} T_{r_{j}}\left(x_{j}\right)\right\rangle_{\beta_{0}}=\left\langle\mathcal{T} \prod_{j}\left(-T_{r_{j}}\left(\mathrm{i} r_{j} x_{j}\right)\right)\right\rangle_{L / v}
$$

as the equilibrium expectations with inverse temperature $L / v$ in the theory on the circle with circumference $v \beta_{0}$. The components of the energy-momentum tensor with complex arguments on the right-hand side are defined by

$$
T_{ \pm}(x \pm \mathrm{i} v \tau)=\mathrm{e}^{\tau H} T_{ \pm}(x) \mathrm{e}^{-\tau H}
$$

and $\mathcal{T}$ orders $x_{j}$ increasingly from the right to the left. The identity in (3.20) comes from swapping the two circles that play a symmetric role in the Euclidean version of the theory. It still holds for the Luttinger model if the finite-volume theory corresponds to antiperiodic boundary conditions for the fermionic fields $\psi_{ \pm}(x)$, as we assumed, even if the corresponding CFT does not have full modular invariance.

When $L \rightarrow \infty$, the right-hand side of (3.20) tends to the vacuum expectations providing the dual representation of the equilibrium expectations in the thermodynamic limit:

$$
\left\langle\prod_{j} T_{r_{j}}\left(x_{j}\right)\right\rangle_{\beta_{0}}^{\infty}=\left\langle 0\left|\mathcal{T} \prod_{j}\left(-T_{r_{j}}\left(\mathrm{i}_{j} x_{j}\right)\right)\right| 0\right\rangle .
$$

Besides, such vacuum expectations are universal because they receive contribution only from the tensor product of the two vacuum highest-weight representations of the Virasoro algebra. They factorize according to

$$
\left\langle 0\left|\mathcal{T} \prod_{j}\left(-T_{r_{j}}\left(\mathrm{i} r_{j} x_{j}\right)\right)\right| 0\right\rangle=\left\langle 0\left|\mathcal{T} \prod_{j: r_{j}=+}\left(-T_{+}\left(\mathrm{i} x_{j}\right)\right)\right| 0\right\rangle\left\langle 0\left|\mathcal{T} \prod_{j: r_{j}=-}\left(-T_{-}\left(-\mathrm{i} x_{j}\right)\right)\right| 0\right\rangle .
$$

In the theory on the circle with circumference $v \beta_{0}$,

$$
\begin{aligned}
\left\langle 0\left|T_{ \pm}\left( \pm \mathrm{i} x_{1}\right)\right| 0\right\rangle & =-\frac{\pi c}{12\left(v \beta_{0}\right)^{2}} \\
\left\langle 0\left|\mathcal{T} T_{ \pm}\left( \pm \mathrm{i} x_{1}\right) T_{ \pm}\left( \pm \mathrm{i} x_{2}\right)\right| 0\right\rangle & =\left(\frac{\pi c}{12\left(v \beta_{0}\right)^{2}}\right)^{2}+\frac{\pi^{2} c}{8\left(v \beta_{0}\right)^{4} \sinh ^{4}\left(\frac{\pi}{v \beta_{0}}\left(x_{1}-x_{2}\right)\right)}
\end{aligned}
$$

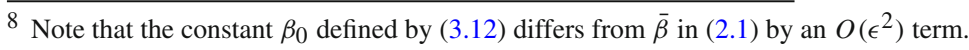




$$
\left\langle 0\left|\mathcal{T} T_{ \pm}\left( \pm \mathrm{i} x_{1}\right) T_{\mp}\left(\mp \mathrm{i} x_{2}\right)\right| 0\right\rangle=\left(\frac{\pi c}{12\left(v \beta_{0}\right)^{2}}\right)^{2} .
$$

We infer that the identity in (3.17) holds in the thermodynamic limit for infinite-volume profiles $\beta(x)>0$ with arbitrary asymptotic values $\beta_{\mathcal{L}}$ and $\beta_{\mathcal{R}}$ and the function $f$ defined by (1.10). By scaling, the right-hand side is then independent of the choice of $\beta_{0}$. In particular, (3.18) together with (3.24) show that in the limit $L \rightarrow \infty$,

$$
\left\langle\left. T_{ \pm}\left(x^{\mp}\right)\right|_{\text {neq }} ^{\infty}=f^{\prime}\left(x^{\mp}\right)^{2} \frac{\pi c}{12\left(v \beta_{0}\right)^{2}}-\frac{c}{24 \pi}(S f)\left(x^{\mp}\right)=\frac{\pi c}{12\left(v \beta\left(x^{\mp}\right)\right)^{2}}-\frac{c}{24 \pi}(S f)\left(x^{\mp}\right) .\right.
$$

This is equivalent to (1.7) with $F$ given by the right-hand side of (1.8) multiplied by the central charge $c$.

The above result allows one to easily extract the value of the thermal Drude weight $D_{\text {th }}$, see (1.16), which may be obtained $[18,48]$ from

$$
D_{\text {th }}=-\beta_{0}^{2} \lim _{\beta_{\mathcal{L}, \mathcal{R}} \rightarrow \beta_{0}} \frac{1}{\Delta \beta} \lim _{t \rightarrow \infty} \frac{1}{t} \int\left\langle\left.\mathcal{J}(x, t)\right|_{\text {neq }} ^{\infty} d x,\right.
$$

where the nonequilibrium expectation is calculated for the inverse-temperature profile $\beta(x)$ interpolating between the asymptotic values $\beta_{\mathcal{L}}$ and $\beta_{\mathcal{R}}$ with $\Delta \beta=\beta_{\mathcal{L}}-\beta_{\mathcal{R}}$. The factor $-\beta_{0}^{2}$ is there to relate $D_{\text {th }}$ to the response to temperature rather than inverse-temperature change. The space integral receives the contribution from the region of length $\approx 2 v t$ between the two ballistically separating heat waves where the heat current takes the long-time value $(\pi c / 12)\left(\beta_{\mathcal{L}}^{-2}-\beta_{\mathcal{R}}^{-2}\right)$. This yields

$$
D_{\mathrm{th}}=\frac{\pi v c}{3 \beta_{0}}
$$

which is proportional to the temperature $1 / \beta_{0}$. The result may be also obtained using the partitioning protocol that leads to the same steady state. It agrees with the calculation of the thermal conductivity by the Green-Kubo formula,

$$
\begin{aligned}
\kappa_{\text {th }}(\omega) & =\beta_{0} \int_{0}^{\infty} \mathrm{e}^{\mathrm{i} \omega t} d t \int_{0}^{\beta_{0}} d \tau \int\left\langle\left.\mathcal{J}(x, t) \mathcal{J}(0, i \tau)\right|_{\beta_{0}} ^{\infty} d x\right. \\
& =\frac{\pi^{2} c}{8 \beta_{0}^{3}} \int_{0}^{\infty} \mathrm{e}^{\mathrm{i} \omega t} d t \int_{0}^{\beta_{0}} d \tau \int \sum_{r= \pm} \sinh ^{-4}\left(\frac{\pi(x-r v t+r \mathrm{i} v \tau)}{v \beta_{0}}\right) d x \\
& =\frac{\pi v c}{4 \beta_{0}} \int_{0}^{\infty} \mathrm{e}^{\mathrm{i} \omega t} d t \int \cosh ^{-4}(y) d y=\frac{\pi v c}{3 \beta_{0}}\left(\pi \delta(\omega)+\mathrm{i} P V \frac{1}{\omega}\right),
\end{aligned}
$$

where we used (3.25) and (3.26) to express the infinite-volume 2-point correlation function of the heat current. Note that the regular part of $\operatorname{Re} \kappa_{\text {th }}(\omega)$ vanishes, confirming the absence of diffusive heat transport in nonequilibrium CFT.

\section{Generalizations}

\subsection{Other Correlators}

The finite-volume relation in (3.15) between the nonequilibrium and equilibrium expectations may be rendered explicit also for observables 


$$
\mathcal{O}=\prod_{j} \Phi_{j}\left(x_{j}^{-}, x_{j}^{+}\right)
$$

where $\Phi_{j}\left(x_{j}^{-}, x_{j}^{+}\right)$are primary fields whose transformation laws under the $\widetilde{\operatorname{Diff}_{+}}\left(S^{1}\right)$ symmetry take the form

$U_{+}(f) U_{-}(f) \Phi_{j}\left(x^{-}, x^{+}\right) U_{-}(f)^{-1} U_{+}(f)^{-1}=f^{\prime}\left(x^{-}\right)^{\Delta_{\Phi_{j}}^{+}} f^{\prime}\left(x^{+}\right)^{\Delta_{\Phi_{j}}^{-}} \Phi_{j}\left(f\left(x^{-}\right), f\left(x^{+}\right)\right)$

with the conformal weights $\Delta_{\Phi_{j}}^{ \pm} \geq 0$. It then follows from (3.15) that

$$
\left\langle\prod_{j} \Phi_{j}\left(x_{j}^{-}, x_{j}^{+}\right)\right\rangle_{\mathrm{neq}}=\left\langle\prod_{j}\left(f^{\prime}\left(x_{j}^{-}\right)^{\Delta_{\Phi_{j}}^{+}} f^{\prime}\left(x_{j}^{+}\right)^{\Delta_{\Phi_{j}}^{-}} \Phi_{j}\left(f\left(x_{j}^{-}\right), f\left(x_{j}^{+}\right)\right)\right)\right\rangle_{\beta_{0}}
$$

for $f$ given by (1.10) and $\beta_{0}$ by (3.12). Note that these are simpler relations than for the energymomentum tensor components $T_{ \pm}$since those fail to be primary fields with conformal weights $(2,0)$ and $(0,2)$ due to the Schwarzian derivative term in (3.7) reflecting the conformal anomaly. In a similar way as for observables built from the operators $T_{ \pm}$, the relations (4.3) hold also in the thermodynamic limit which may be controlled like before. Also, as before, $\beta_{0}$ may be taken arbitrary in the infinite volume.

For example, the Luttinger model has a conserved $\mathrm{U}(1)$ current with the light-cone components $J_{ \pm}\left(x^{\mp}\right)=\sqrt{K} \tilde{\rho}_{ \pm}\left(x^{\mp}\right)$, see $(3.5)$, with conformal weights $(1,0)$ and $(0,1)$, respectively, and (renormalized) fermionic fields $\psi_{ \pm}\left(x^{-}, x^{+}\right)$with conformal weights

$$
\left(\Delta_{\psi_{+}}^{+}, \Delta_{\psi_{+}}^{-}\right)=\left(\frac{(K+1)^{2}}{8 K}, \frac{(K-1)^{2}}{8 K}\right), \quad\left(\Delta_{\psi_{-}}^{+}, \Delta_{\psi_{-}}^{-}\right)=\left(\frac{(K-1)^{2}}{8 K}, \frac{(K+1)^{2}}{8 K}\right)
$$

accompanied by their Hermitian conjugates $\psi_{ \pm}^{\dagger}\left(x^{-}, x^{+}\right)$with the same conformal weights. ${ }^{9}$ Their infinite-volume equilibrium 2-point correlation functions have the form

$$
\left\langle\left. J_{ \pm}\left(x^{\mp}\right) J_{ \pm}\left(y^{\mp}\right)\right|_{\beta_{0}} ^{\infty}=-\frac{K}{4\left(v \beta_{0}\right)^{2} \sinh ^{2}\left(\frac{\pi}{v \beta_{0}}\left(x^{\mp}-y^{\mp}\right)\right)}\right.
$$

and

$$
\begin{aligned}
& \left\langle\left.\psi_{ \pm}\left(x^{-}, x^{+}\right) \psi_{ \pm}^{\dagger}\left(y^{-}, y^{+}\right)\right|_{\beta_{0}} ^{\infty}\right. \\
& =\frac{\left.\mathrm{e}^{\pi \mathrm{i}\left[\Delta_{\psi_{ \pm}}^{+} \operatorname{sgn}\left(x^{-}-y^{-}\right)-\Delta_{\psi_{ \pm}}^{-}\right.} \operatorname{sgn}\left(x^{+}-y^{+}\right)\right]}{2 \pi\left(\frac{v \beta_{0}}{\pi} \sinh \left(\frac{\pi}{v \beta_{0}}\left|x^{-}-y^{-}\right|\right)\right)^{2 \Delta_{\psi_{ \pm}}^{+}}\left(\frac{v \beta_{0}}{\pi} \sinh \left(\frac{\pi}{v \beta_{0}}\left|x^{+}-y^{+}\right|\right)\right)^{2 \Delta_{\psi_{ \pm}}^{-}}} .
\end{aligned}
$$

Thus, it follows from (4.3) that the corresponding nonequilibrium correlation functions are

$$
\left\langle\left. J_{ \pm}\left(x^{\mp}\right) J_{ \pm}\left(y^{\mp}\right)\right|_{\text {neq }} ^{\infty}=-\frac{K}{4 v^{2} \beta\left(x^{\mp}\right) \beta\left(y^{\mp}\right) \sinh ^{2}\left(\int_{y^{\mp}}^{x^{\mp}} \frac{\pi}{v \beta\left(x^{\prime}\right)} d x^{\prime}\right)}\right.
$$

\footnotetext{
9 The fermionic fields are represented as vertex operators related to the bosonic fields $\tilde{\rho}_{ \pm}$. Such operators require Wick ordering that provides their multiplicative renormalization, see, e.g., [38].
} 
and

$$
\begin{aligned}
& \left\langle\left.\psi_{ \pm}\left(x^{-}, x^{+}\right) \psi_{ \pm}^{\dagger}\left(y^{-}, y^{+}\right)\right|_{\text {neq }} ^{\infty}\right.
\end{aligned}
$$

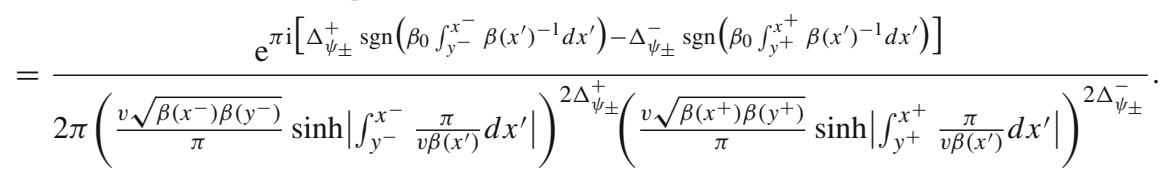

We note that the latter agrees with Eq. (19) in [33] to first order in the expansion parameter $\epsilon$ in (2.1) and exactly reproduces Eq. (10) in [33] in the long-time limit.

\subsection{Temperature and Velocity Profiles}

It is straightforward to generalize the argument of Sect. 3.2 to nonequilibrium states as in (1.3) with $\mathcal{G}$ given by (1.12), which is more conveniently written as

$$
\mathcal{G}=v \int_{-L / 2}^{L / 2}\left[\beta_{+}(x) T_{+}(x)+\beta_{-}(x) T_{-}(x)\right] d x, \quad \beta_{ \pm}(x)=\beta(x)[1 \pm v(x) / v] .
$$

Our argument above goes through as it stands but with $U(f)$ replaced by $U\left(f_{+}, f_{-}\right)=$ $U_{+}\left(f_{+}\right) U_{-}\left(f_{-}\right)$with two different diffeomorphisms $f_{ \pm}$. Choosing them as

$$
f_{ \pm}(x)=\int_{0}^{x} \frac{\beta_{0, \pm}}{\beta_{ \pm}\left(x^{\prime}\right)} d x^{\prime}, \quad \frac{1}{\beta_{0, \pm}}=\frac{1}{L} \int_{-L / 2}^{L / 2} \frac{1}{\beta_{ \pm}(x)} d x,
$$

one straightens out both profiles $1 / \beta_{ \pm}(x)$, replacing (3.15) by the identity

$$
\langle\mathcal{O}\rangle_{\text {neq }}=\left\langle U\left(f_{+}, f_{-}\right) \mathcal{O} U\left(f_{+}, f_{-}\right)^{-1}\right\rangle_{\beta_{0,+}, \beta_{0,-}},
$$

where

$$
\langle\mathcal{O}\rangle_{\beta_{0,+}, \beta_{0,-}}=\frac{\operatorname{Tr}\left(\mathrm{e}^{-\beta_{0,+} H_{+}-\beta_{0,-} H_{-}} \mathcal{O}\right)}{\operatorname{Tr}\left(\mathrm{e}^{-\beta_{0,+} H_{+}-\beta_{0,-} H_{-}}\right)}, \quad H_{ \pm}=v \int_{-L / 2}^{L / 2} T_{ \pm}(x) d x,
$$

define the expectations in a simple example of a generalized Gibbs state with different temperatures for the right and the left movers. In particular, (3.17) becomes

$$
\left\langle\prod_{j} T_{r_{j}}\left(x_{j}^{-r_{j}}\right)\right\rangle_{\text {neq }}=\left\langle\prod_{j}\left(f_{r_{j}}^{\prime}\left(x_{j}^{-r_{j}}\right)^{2} T_{r_{j}}\left(f_{r_{j}}\left(x_{j}^{-r_{j}}\right)\right)-\frac{c}{24 \pi}\left(S f_{r_{j}}\right)\left(x_{j}^{-r_{j}}\right)\right)\right\rangle_{\beta_{0,+}, \beta_{0,-}} .
$$

The thermodynamic limit of the expectations in (4.12) of the observables in (3.16) may still be conveniently studied by going to the dual picture which, upon setting $\beta_{0, \pm}=\beta_{0}\left(1 \pm v_{0} / v\right)$, takes the form ${ }^{10}$

$$
\left\langle\prod_{j} T_{r_{j}}\left(x_{j}\right)\right\rangle_{\beta_{0,+}, \beta_{0,-}}=\left\langle\mathcal{T} \prod_{j}\left[-\frac{1}{\left(1+r_{j} v_{0} / v\right)^{2}} T_{r_{j}}\left(\mathrm{i} r_{j} \frac{x_{j}}{\left(1+r_{j} v_{0} / v\right)}\right)\right]\right\rangle_{L}, \frac{L}{v+v_{0}},
$$

where on the right-hand side the expectation is in the theory on the circle with circumference $v \beta_{0}$. We infer that in the thermodynamic limit

10 This is proven using modular invariance for imaginary $v_{0}$ and continuing analytically to real $v_{0}$. 


$$
\begin{aligned}
\left\langle\prod_{j} T_{r_{j}}\left(x_{j}\right)\right\rangle_{\beta_{0,+}, \beta_{0,-}}^{\infty} & =\left\langle 0\left|\mathcal{T} \prod_{j}\left[-\frac{1}{\left(1+r_{j} v_{0} / v\right)^{2}} T_{r_{j}}\left(\mathrm{i} r_{j} \frac{x_{j}}{\left(1+r_{j} v_{0} / v\right)}\right)\right]\right| 0\right\rangle \\
& =\left\langle 0\left|\mathcal{T} \prod_{j: r_{j}=+}\left(-T_{+}\left(\mathrm{i} x_{j}\right)\right)\right| 0\right\rangle\left\langle 0\left|\mathcal{T} \prod_{j: r_{j}=-}\left(-T_{-}\left(-\mathrm{i} x_{j}\right)\right)\right| 0\right\rangle,
\end{aligned}
$$

where on the right-hand side the first (second) vacuum expectation is in the theory on the circle with circumference $v \beta_{0,+}\left(v \beta_{0,-}\right)$, and the last equality follows by the factorization in (3.23) of the vacuum expectations and their rescaling. In a similar way as in Sect. 3.3, this shows that the identity in (4.13) holds in the thermodynamic limit with the infinite-volume profiles $\beta_{ \pm}(x)>0$ with arbitrary positive asymptotic values $\beta_{ \pm, \mathcal{L}}$ and $\beta_{ \pm, \mathcal{R}}$ and $f_{ \pm}(x)$ given by the first of the equations in (4.10) with arbitrary $\beta_{0, \pm}>0$. In particular,

$$
\left\langle\left. T_{ \pm}\left(x^{\mp}\right)\right|_{\text {neq }} ^{\infty}=\frac{\pi c}{12\left(v \beta_{ \pm}\left(x^{\mp}\right)\right)^{2}}-\frac{c}{24 \pi}\left(S f_{ \pm}\right)\left(x^{\mp}\right)\right.
$$

implying that

$$
\left\langle\left.\mathcal{E}(x, t)\right|_{\text {neq }} ^{\infty}=\frac{1}{2}\left[F_{+}\left(x^{-}\right)+F_{-}\left(x^{+}\right)\right], \quad\left\langle\mathcal { J } \left( x,\left.t\right|_{\text {neq }} ^{\infty}=\frac{v}{2}\left[F_{+}\left(x^{-}\right)-F_{-}\left(x^{+}\right)\right]\right.\right.\right.
$$

with the functions

$$
F_{ \pm}(x)=\frac{\pi c}{6 v} \frac{1}{\beta_{ \pm}(x)^{2}}+\frac{v c}{12 \pi}\left(\frac{\beta_{ \pm}^{\prime \prime}(x)}{\beta_{ \pm}(x)}-\frac{1}{2}\left(\frac{\beta_{ \pm}^{\prime}(x)}{\beta_{ \pm}(x)}\right)^{2}\right),
$$

as described in Sect. 1.

There is a conceptual gain from the consideration of the nonequilibrium states with different temperature profiles for the right and left movers: unlike the states with equal profiles, such states are preserved by the Schrödinger-picture dynamics. Indeed, for $\mathcal{G}$ given by (4.9),

$$
\mathrm{e}^{-\mathrm{i} t H} \mathcal{G} \mathrm{e}^{\mathrm{i} t H}=v \int_{-L / 2}^{L / 2}\left[\beta_{+}\left(x^{-}\right) T_{+}(x)+\beta_{-}\left(x^{+}\right) T_{-}(x)\right] d x
$$

so that under the Schrödinger-picture evolution the profiles $\beta_{ \pm}(x)$ move ballistically to the right and to the left, respectively. This still holds in the thermodynamic limit and makes it clear why for long times such states converge to the generalized Gibbs state in (4.12) with inverse temperatures $\beta_{0,+}=\beta_{+, \mathcal{L}}$ and $\beta_{0,-}=\beta_{-, \mathcal{R}}$. Conversely, we may view the nonequilibrium states with $\mathcal{G}$ given by (4.9) as the generalized Gibbs state with local profiles $\beta_{ \pm}(x)$ whose time evolution under the Schrödinger-picture dynamics reduces to the time evolution of the profiles governed by the equations

$$
\partial_{t} \beta_{ \pm} \pm v \partial_{x} \beta_{ \pm}=0
$$

This is reminiscent of the time evolution of the generalized Gibbs states with local profiles in the generalized hydrodynamic picture of integrable models out of equilibrium [14-18,2023]. In CFT, however, no hydrodynamic-scale closure is needed to obtain the hydrodynamic evolution equation (4.20) [49].

\subsection{Temperature and Chemical-Potential Profiles}

Suppose that our CFT possesses a U(1) symmetry generated by a conserved current with components $\rho$ and $j$ satisfying

$$
\partial_{t} \rho+\partial_{x} j=0
$$


For instance, $\rho=\rho_{+}+\rho_{-}$and $j=K v\left(\rho_{+}-\rho_{-}\right)$for the local Luttinger model, see, e.g., Appendix B in [36] (we recall that $K$ is the Luttinger parameter). One may then consider states with both temperature and chemical-potential ${ }^{11}$ profiles by taking

$$
\mathcal{G}=\int_{-L / 2}^{L / 2} \beta(x)[\mathcal{E}(x)-\mu(x) \rho(x)] d x,
$$

where $\rho(x)$ is the zero time density and $\mu(x)$ is a periodic chemical-potential profile. Suppose that the light-cone components $J_{ \pm}=(1 / 2)\left(\rho \pm v^{-1} j\right)$ of the current depend only on $x^{\mp}$, respectively, and satisfy the $\mathrm{U}(1)$ current algebra:

$$
\begin{array}{ll}
{\left[J_{ \pm}(x), J_{ \pm}(y)\right]= \pm \frac{\kappa}{2 \pi \mathrm{i}} \delta^{\prime}(x-y),} & {\left[J_{ \pm}(x), J_{\mp}(y)\right]=0,} \\
{\left[T_{ \pm}(x), J_{ \pm}(y)\right]=\mp \mathrm{i} \delta^{\prime}(x-y) J_{ \pm}(y) \pm \mathrm{i} \delta(x-y) J_{ \pm}^{\prime}(y),} & {\left[T_{ \pm}(x), J_{\mp}(y)\right]=0 .}
\end{array}
$$

In terms of the Fourier modes,

$$
J_{ \pm}(x)=\frac{1}{L} \sum_{n=-\infty}^{\infty} \mathrm{e}^{ \pm \frac{2 \pi \mathrm{i} n x}{L}} J_{n}^{ \pm}
$$

and the commutation relations take the form

$$
\left[J_{n}^{ \pm}, J_{m}^{ \pm}\right]=\kappa n \delta_{n+m, 0}, \quad\left[J_{n}^{ \pm}, J_{m}^{\mp}\right]=0, \quad\left[L_{n}^{ \pm}, J_{m}^{ \pm}\right]=-m J_{n+m}^{ \pm}, \quad\left[L_{n}^{ \pm}, J_{m}^{\mp}\right]=0 .
$$

For concreteness, we shall restrict our discussion to the case of the local Luttinger model in which case $J_{ \pm}=\sqrt{K} \tilde{\rho}_{ \pm}$and $\kappa=K$, see (3.5), or to the case when $J_{ \pm}$is one of the Cartan subalgebra components of the two current algebras of the level $k$ WZW theory based on a compact Lie group [40] (e.g., $\mathrm{U}(1)$ or $\mathrm{SU}(N))$ in which case $\kappa=k / 2$. It follows from $[47,50]$ that in these cases there exist, besides the two projective representations $U_{ \pm}$of $\widetilde{\operatorname{Diff}}+\left(S^{1}\right)$ considered above for which

$$
U_{ \pm}(f) J_{ \pm}(x) U_{ \pm}(f)^{-1}=f^{\prime}(x) J_{ \pm}(f(x)), \quad U_{ \pm}(f) J_{\mp}(x) U_{ \pm}(f)^{-1}=J_{\mp}(x),
$$

also two commuting projective representations $V_{ \pm}$of the additive gauge group of periodic smooth maps $h(x)=h(x+L)$ on $\mathbb{R}$ generated infinitesimally by $J_{ \pm}$,

$$
V_{ \pm}(h)=I \mp \mathrm{i} \varepsilon \int_{-L / 2}^{L / 2} \xi(x) J_{ \pm}(x) d x+o(\varepsilon)
$$

for $h(x)=\varepsilon \xi(x)$. Under the adjoint action of $V_{ \pm}(h)$,

$$
\begin{array}{lll}
V_{ \pm}(h) J_{ \pm}(x) V_{ \pm}(h)^{-1} & =J_{ \pm}(x)+\frac{\kappa}{2 \pi} h^{\prime}(x), & V_{ \pm}(h) J_{\mp}(x) V_{ \pm}(h)^{-1}=J_{\mp}(x), \\
V_{ \pm}(h) T_{ \pm}(x) V_{ \pm}(h)^{-1}=T_{ \pm}(x)+h^{\prime}(x) J_{ \pm}(x)+\frac{\kappa}{4 \pi} h^{\prime}(x)^{2}, & V_{ \pm}(h) T_{\mp}(x) V_{ \pm}(h)^{-1}=T_{\mp}(x) .
\end{array}
$$

Recall from Sect. 3.2 that the conjugation with operators $U(f)=U_{+}(f) U_{-}(f)$ for $f \in \widetilde{\operatorname{Diff}}\left(S^{1}\right)$ satisfying (1.10) with $\beta_{0}$ given by (3.12) was previously used to straighten out

11 The name is somewhat conventional. If $\rho$ is charge density, rather than the particle density, then $-\mu(x)$ would be the electric potential. 
the periodic inverse-temperature profile $\beta(x)$ to a constant one given by $\beta_{0}$. We shall keep the function $f$ as before and choose

$$
h(x)=\frac{1}{v} \int_{0}^{x}\left(\mu\left(x^{\prime}\right)-\frac{\beta_{0}}{\beta\left(x^{\prime}\right)} \mu_{0}\right) d x^{\prime}, \quad \mu_{0}=\frac{1}{L} \int_{-L / 2}^{L / 2} \mu(x) d x .
$$

Then conjugating $\mathcal{G}$ in (4.22) first with $V(h)=V_{+}(h) V_{-}(h)$ and then with $U(f)$ straightens out the chemical-potential and inverse-temperature profiles to $\mu_{0}$ and $\beta_{0}$, respectively,

$$
U(f) V(h) \mathcal{G} V(h)^{-1} U(f)^{-1}=\int_{-L / 2}^{L / 2} \beta_{0}\left[\mathcal{E}(y)-\mu_{0} \rho(y)\right] d y+\text { const },
$$

leading to the identity

$$
\langle\mathcal{O}\rangle_{\text {neq }}=\left\langle U(f) V(h) \mathcal{O} V(h)^{-1} U(f)^{-1}\right\rangle_{\beta_{0}, \mu_{0}} .
$$

The thermodynamic limit can be controlled as before using the dual representation of the equilibrium expectations (that involves now the theory on a circle of circumference $v \beta_{0}$ with twisted boundary conditions). In the infinite volume, one can again treat profiles with arbitrary asymptotic values. Using the fact that in the theories under consideration,

$$
\left\langle\left. T_{ \pm}(x)\right|_{\beta_{0}, \mu_{0}} ^{\infty}=\frac{\pi c}{12\left(v \beta_{0}\right)^{2}}+\frac{\kappa \mu_{0}^{2}}{4 \pi v^{2}}, \quad\left\langle\left. J_{ \pm}(x)\right|_{\beta_{0}, \mu_{0}} ^{\infty}=\frac{\kappa \mu_{0}}{2 \pi v}\right.\right.
$$

in the thermodynamic limit, one obtains the identities in (1.7) with

$$
F(x)=\frac{\pi c}{6 v} \frac{1}{\beta(x)^{2}}+\frac{c v}{12 \pi}\left(\frac{\beta^{\prime \prime}(x)}{\beta(x)}-\frac{1}{2}\left(\frac{\beta^{\prime}(x)}{\beta(x)}\right)^{2}\right)+\frac{\kappa}{2 \pi v} \mu(x)^{2}
$$

as well as the formulas

$$
\left\langle\left.\rho(x, t)\right|_{\text {neq }} ^{\infty}=\frac{1}{2}\left[G\left(x^{-}\right)+G\left(x^{+}\right)\right], \quad\left\langle\left. j(x, t)\right|_{\text {neq }} ^{\infty}=\frac{v}{2}\left[G\left(x^{-}\right)-G\left(x^{+}\right)\right]\right.\right.
$$

with

$$
G(x)=\frac{\kappa}{\pi v} \mu(x) .
$$

For the matrix $\mathcal{D}$ of the Drude weights obtained from the nonequilibrium expectations with respect to states with profiles with small kinks of heights $\Delta \beta=\beta_{\mathcal{L}}-\beta_{\mathcal{R}}$ and $\Delta \mu=\mu_{\mathcal{L}}-\mu_{\mathcal{R}}$ around the constant values $\beta_{0}$ and $\mu_{0}$ of the inverse temperature and chemical potential, the formula in (3.28) generalizes to

$$
\begin{aligned}
& \mathcal{D}=\left(\begin{array}{ll}
D_{11} & D_{12} \\
D_{21} & D_{22}
\end{array}\right)
\end{aligned}
$$

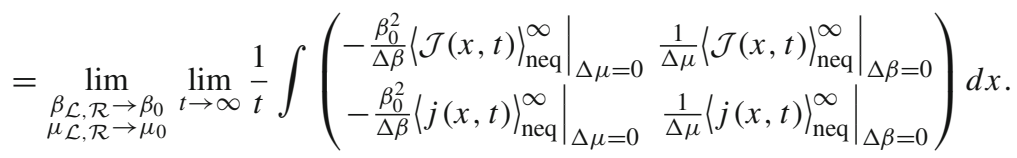

This yields

$$
\mathcal{D}=\left(\begin{array}{ll}
\frac{\pi v c}{3 \beta_{0}} & \frac{\kappa v \mu_{0}}{\pi} \\
0 & \frac{\kappa v}{\pi}
\end{array}\right) .
$$

Note that the thermal Drude weight $D_{11}$ is independent of $\mu_{0}$ and the density Drude weight $D_{22}$ is independent of the temperature. In particular, for the Luttinger model, $D_{22}=K v / \pi$. 
The lack of symmetry of $\mathcal{D}$ is due to the asymmetric way in which the temperature and the chemical potential enter into the Gibbs state. ${ }^{12}$

As before, one may also consider nonequilibrium states with different local profiles $\beta_{ \pm}(x)$ and $\mu_{ \pm}(x)$ for right and left movers, which are defined by (1.3) with

$$
\mathcal{G}=\int_{-L / 2}^{L / 2} \sum_{r= \pm} \beta_{r}(x)\left[v T_{r}(x)-\mu_{r}(x) J_{r}(x)\right] d x .
$$

This leads to the replacement of the functions $F$ and $G$ in (4.35) and (4.37) with

$$
F_{ \pm}(x)=\frac{\pi c}{6 v} \frac{1}{\beta_{ \pm}(x)^{2}}+\frac{c v}{12 \pi}\left(\frac{\beta_{ \pm}^{\prime \prime}(x)}{\beta_{ \pm}(x)}-\frac{1}{2}\left(\frac{\beta_{ \pm}^{\prime}(x)}{\beta_{ \pm}(x)}\right)^{2}\right)+\frac{\kappa}{2 \pi v} \mu_{ \pm}(x)^{2}
$$

and

$$
G_{ \pm}(x)=\frac{\kappa}{\pi v} \mu_{ \pm}(x)
$$

respectively. In this case, the expectation values are given by (4.17) for $\left\langle\left.\mathcal{E}(x, t)\right|_{\text {neq }} ^{\infty}\right.$ and $\left\langle\left.\mathcal{J}(x, t)\right|_{\text {neq }} ^{\infty}\right.$ as well as

$$
\left\langle\left.\rho(x, t)\right|_{\text {neq }} ^{\infty}=\frac{1}{2}\left[G_{+}\left(x^{-}\right)+G_{-}\left(x^{+}\right)\right], \quad\left\langle\left. j(x, t)\right|_{\text {neq }} ^{\infty}=\frac{v}{2}\left[G_{+}\left(x^{-}\right)-G_{-}\left(x^{+}\right)\right] .\right.\right.
$$

The nonequilibrium states in (4.40) form the family of generalized Gibbs states with local profiles that correspond to the commuting conserved charges $H_{ \pm}$of (4.12) and $Q_{ \pm}^{J}=\int_{-L / 2}^{L / 2} J_{ \pm}(x) d x$. Such a family of states is again preserved by the Schrödinger evolution that displaces the local profiles $\beta_{ \pm}(x)$ and $\mu_{ \pm}(x)$ ballistically. Their time evolutions are governed by (4.20) together with

$$
\partial_{t} \mu_{ \pm} \pm v \partial_{x} \mu_{ \pm}=0
$$

In the long-time limit, one obtains a genuine (i.e., with constant profiles) generalized Gibbs state which is the thermodynamic limit of the state with

$$
\mathcal{G}=\beta_{+, \mathcal{L}}\left(H_{+}-\mu_{+, \mathcal{L}} Q_{+}^{J}\right)+\beta_{-, \mathcal{R}}\left(H_{-}-\mu_{-, \mathcal{R}} Q_{-}^{J}\right) .
$$

In particular,

$$
\begin{aligned}
& \lim _{t \rightarrow \infty}\langle\mathcal{E}(x, t)\rangle_{\text {neq }}^{\infty}=\frac{\pi c}{12 v}\left(\beta_{+, \mathcal{L}}^{-2}+\beta_{-, \mathcal{R}}^{-2}\right)+\frac{\kappa}{4 \pi v}\left(\mu_{+, \mathcal{L}}^{2}+\mu_{-, \mathcal{R}}^{2}\right), \\
& \lim _{t \rightarrow \infty}\langle\mathcal{J}(x, t)\rangle_{\text {neq }}^{\infty}=\frac{\pi c}{12}\left(\beta_{+, \mathcal{L}}^{-2}-\beta_{-, \mathcal{R}}^{-2}\right)+\frac{\kappa}{4 \pi}\left(\mu_{+, \mathcal{L}}^{2}-\mu_{-, \mathcal{R}}^{2}\right), \\
& \lim _{t \rightarrow \infty}\langle\rho(x, t)\rangle_{\text {neq }}^{\infty}=\frac{\kappa}{2 \pi v}\left(\mu_{+, \mathcal{L}}+\mu_{-, \mathcal{R}}\right), \\
& \lim _{t \rightarrow \infty}\langle j(x, t)\rangle_{\text {neq }}^{\infty}=\frac{\kappa}{2 \pi}\left(\mu_{+, \mathcal{L}}-\mu_{-, \mathcal{R}}\right)
\end{aligned}
$$

generalizing (1.15).

As a specific example, consider the Luttinger model with the initial state given by (4.40) with $\beta_{ \pm}(x)=\beta_{0}$ and with $\mu_{ \pm}(x)$ interpolating when $L \rightarrow \infty$ between the asymptotic values $\mu_{ \pm, \mathcal{L}}$ and $\mu_{ \pm, \mathcal{R}}$. The charges $Q_{ \pm}^{J}=\sqrt{K} \int \tilde{\rho}_{ \pm}(x) d x$ are then the number operators of right and left moving plasmons. One can also consider in that case the number operators

12 The coefficients of linear response of currents $\mathcal{J}$ and $j$ to small $-\Delta \beta$ and $\Delta(\beta \mu)$ would form a symmetric matrix that unveils a ballistic version of the Onsager reciprocal relations. 
of electrons (and holes) $Q_{ \pm}^{e}=\int \rho_{ \pm}(x) d x$, which are different from $Q_{ \pm}^{J}$, although the total charges are equal, i.e., $Q_{+}^{e}+Q_{-}^{e}=Q_{+}^{J}+Q_{-}^{J}$. Indeed, we infer from (3.5) that

$$
Q_{ \pm}^{J}=\frac{K+1}{2} Q_{ \pm}^{e}-\frac{K-1}{2} Q_{\mp}^{e} .
$$

Unlike for $Q_{ \pm}^{J}$, the spectra of $Q_{ \pm}^{e}$ are composed of integers. In terms of $Q_{ \pm}^{e}$, the generalized Gibbs state appearing in the long-time limit of the evolution will correspond to

$$
\mathcal{G}=\beta_{0}\left(H-\mu_{+}^{e} Q_{+}^{e}-\mu_{-}^{e} Q_{-}^{e}\right), \quad \mu_{ \pm}^{e}=\frac{1}{2}\left(\mu_{+, \mathcal{L}}+\mu_{-, \mathcal{R}}\right) \pm \frac{K}{2}\left(\mu_{+, \mathcal{L}}-\mu_{-, \mathcal{R}}\right) .
$$

From (4.49) we infer that the value of the permanent current in the limiting nonequilibrium steady state is

$$
I=\frac{K}{2 \pi}\left(\mu_{+, \mathcal{L}}-\mu_{-, \mathcal{R}}\right)=\frac{1}{2 \pi}\left(\mu_{+}^{e}-\mu_{-}^{e}\right) .
$$

It was argued in [51-53] that $\mu_{ \pm}^{e}$ that couple in the steady state to the electron charges correspond to the chemical potentials of free electrons of wide leads connected to a Luttinger wire, at least if $\mu_{+}(x)=\mu_{-}(x)$. As was discussed in [36], the second equality of (4.52) would then provide an explanation for the experimental measurements [54] of conductance in quantum wires that gave results close to the universal constant $e^{2} / h$, equal to $1 / 2 \pi$ in the units $\hbar=e=1$ that we are using. This universal value is different from the conductance $K e^{2} / h$ predicted in [55] which, instead, is consistent with the first equality of (4.52) that uses the asymptotic values of the imposed chemical-potential profiles that couple in the steady state to the plasmon charges. The above extends the derivation of the universal result obtained in [36] to states with constant temperature and with chemical-potential profiles possibly different for the right and left movers. ${ }^{13}$

The Luttinger model possesses also a conserved axial current with $\rho_{A}=\rho_{+}-\rho_{-}$and $j_{A}=(v / K)\left(\rho_{+}+\rho_{-}\right)$satisfying $\partial_{t} \rho_{A}+\partial_{x} j_{A}=0$. Note that (4.48) implies that the permanent axial current in the limiting nonequilibrium steady state considered above takes the value

$$
I_{A}=\frac{1}{2 \pi}\left(\mu_{+, \mathcal{L}}+\mu_{-, \mathcal{R}}\right)=\frac{1}{2 \pi}\left(\mu_{+}^{e}+\mu_{-}^{e}\right)
$$

with the universal coefficient both when expressed in terms of the asymptotic values of the profiles $\mu_{ \pm}(x)$ and in terms of $\mu_{ \pm}^{e}$.

\section{Equilibrium Dynamics and Relation to Euclidian CFT}

In this section, we discuss the relation between the articles [29-32] and the present paper. In [29] it was argued that the kernel of the 1-particle density matrix in the ground state of a nonrelativistic high density Fermi gas in a trap may be described on mesoscopic scales by the 2-point function of the fermionic massless free field whose Fermi velocity varies in space. These results were generalized in [30-32] to certain nonrelativistic systems of interacting $1 d$ bosons in traps. Despite similarities, there are several differences with the approach of the present paper. First, the arguments in [29] were based on the analysis of the ground-state Euclidiantime correlators in the presence of a trap and these were shown to correspond to Euclidian CFT correlators in an appropriate curved metric (in [32] also the coupling to a gauge field

13 The result in [36] was more general in that it was for the Luttinger model with nonlocal interactions, but it was only for zero temperature states and $\mu_{+}(x)=\mu_{-}(x)=\mu(x)$. 
appeared implicitly). In this paper, we consider positive temperatures, but the correspondence of [29] generalizes to low-temperature states leading to the compactification of the Euclidian time direction in CFT, just as for homogeneous equilibria. Hence, for specific CFTs, the states in (1.3) may, indeed, be viewed as describing on mesoscopic scales the $1 d$ nonrelativistic lowtemperature matter in traps, with $\beta(x)$ having the interpretation of the position-dependent Fermi velocity in appropriate units. Second, the argument of [29] was done for the equilibrium dynamics (although some nonequilibrium situations were also considered), whereas in the bulk of the present paper we study the dynamics generated by the homogeneous Hamiltonian that does not preserve the states in (1.3). Our considerations may, however, be generalized to dynamics induced by inhomogeneous Hamiltonians [56], in particular to the ones that preserve the states in (1.3). Third, we use the Minkowski version of CFT, whereas the papers [29-32] employed the Euclidian CFT. That is usually considered as an innocent distinction handled by the Wick rotation. Indeed, for the primary fields, the correlators in the Euclidian theory in the metric considered in [29-32] and with compactified time do agree, up to the Wick rotation, with the corresponding correlators in the states of (1.3) for which the time dependence is generated by the equilibrium dynamics. As shown below, however, that does not hold directly for the correlators of the energy-momentum tensor components which are of main interest in this paper. This points to the need of caution when one applies Euclidian techniques in the study of systems that are inhomogeneous in space or/and time.

To be more concrete, let us briefly discuss the equilibrium dynamics for the states in (1.3) that is generated by the inhomogeneous Hamiltonian $\tilde{H}=\beta_{0}^{-1} \mathcal{G}$. Defining

$$
T_{ \pm}(x ; t)=\mathrm{e}^{\mathrm{i} t \tilde{H}} T_{ \pm}(x) \mathrm{e}^{-\mathrm{i} t \tilde{H}}, \quad \Phi_{j}(x ; t)=\mathrm{e}^{\mathrm{i} t \tilde{H}} \Phi_{j}(x, x) \mathrm{e}^{-\mathrm{i} t \tilde{H}},
$$

we immediately obtain from (3.15) and (3.11) the relations

$$
\begin{aligned}
& \left\langle\prod_{j} T_{r_{j}}\left(x_{j} ; t_{j}\right)\right\rangle_{\text {neq }}=\left\langle\prod_{j}\left(f^{\prime}\left(x_{j}\right)^{2} T_{r_{j}}\left(f\left(x_{j}\right)^{-r_{j}}\right)-\frac{c}{24 \pi}(S f)\left(x_{j}\right)\right)\right\rangle_{\beta_{0}}, \\
& \left\langle\prod_{j} \Phi_{j}\left(x_{j} ; t_{j}\right)\right\rangle_{\text {neq }}=\left\langle\prod_{j}\left(f^{\prime}\left(x_{j}\right)^{\Delta_{\Phi_{j}}^{+}} f^{\prime}\left(x_{j}\right)^{\Delta_{\Phi_{j}}^{-}} \Phi_{j}\left(f\left(x_{j}\right)^{-}, f\left(x_{j}\right)^{+}\right)\right)\right\rangle_{\beta_{0}},
\end{aligned}
$$

where $f\left(x_{j}\right)^{-r}=f\left(x_{j}\right)-r v t_{j}$. Note the difference of the right-hand sides with those of (3.17) and (4.3) corresponding to the dynamics generated by the homogeneous Hamiltonian $H$ of (3.8) which results in the time dependence in the arguments of function $f$.

After the Wick rotation $t_{j}=-i \tau_{j}$, the correlators in (5.3) become the correlation functions of the same primary fields in the Euclidian CFT on the torus $S^{1} \times S^{1}$ parameterized by $\left(x \bmod L, \tau \bmod \beta_{0}\right)$ and equipped with the Riemannian metric

$$
h=(d x)^{2}+\left(v \beta(x) / \beta_{0}\right)^{2}(d \tau)^{2}=\mathrm{e}^{\sigma(z, \bar{z})} h_{0},
$$

where $h_{0}=d z d \bar{z}$ for the complex coordinate $z=f(x)+\mathrm{i} v \tau$ on the torus with $f(x)$ given by (1.10) and $\beta_{0}$ by (3.12), and where $\sigma(z, \bar{z})=-2 \ln f^{\prime}(x)$. In other words,

$$
\left\langle\prod_{j} \Phi_{j}\left(x_{j},-i \tau_{j}\right)\right\rangle_{\mathrm{neq}}=\left\langle\prod_{j} \Phi_{j}\left(z_{j}, \bar{z}_{j}\right)\right\rangle_{S^{1} \times S^{1}, h^{\prime}},
$$

where on the left-hand side is the Wick rotated (5.3) and on the right-hand side the Euclidian correlation functions on the torus $S^{1} \times S^{1}$ with the Riemannian metric $h$ of (5.4). Such a relation is well known for $f(x)=x$. Its generalization to general $f$ follows from the identity

$$
\left\langle\prod_{j} \Phi_{j}\left(z_{j}, \bar{z}_{j}\right)\right\rangle_{S^{1} \times S^{1}, \mathrm{e}^{\sigma} h_{0}}=\left\langle\prod_{j}\left(e^{\frac{1}{2}\left(\Delta_{\Phi_{j}}^{+}+\Delta_{\Phi_{j}}^{-}\right) \sigma\left(z_{j}, \bar{z}_{j}\right)} \Phi_{j}\left(z_{j}, \bar{z}_{j}\right)\right)\right\rangle_{S^{1} \times S^{1}, h_{0}} .
$$


The ground-state version of the relations in (5.5) provided the basis for the use of Euclidian CFT in the description of the trapped $1 d$ fermions or bosons on mesoscopic scales in [29-32], with the interpretation of $v \beta(x) / \beta_{0}$ as the position-dependent Fermi velocity clearly reflected in the form of the metric in (5.4).

Let us pass to the discussion of the energy-momentum correlators. For $f(x)=x$, the Wick-rotated correlators in (5.2) are represented by the Euclidian correlation functions of the energy-momentum components $T_{+}=T_{z z}\left\|(d z)^{2}\right\|$ and $T_{-}=T_{\bar{z} \bar{z}}\left\|(d \bar{z})^{2}\right\|$ on the torus $S^{1} \times S^{1}$ with metric $h_{0}$. However, for general $f(x)$ one has [57], in the notation $z^{+}=z, z^{-}=\bar{z}$,

$$
\begin{aligned}
& \left\langle\prod_{j} T_{r_{j}}\left(z_{j}, \bar{z}_{j}\right)\right\rangle_{S^{1} \times S^{1}, \mathrm{e}^{\sigma} h_{0}} \\
& =\left\langle\prod_{j}\left(e^{-\sigma\left(z_{j}, \bar{z}_{j}\right)}\left(T_{r_{j}}-\frac{c}{24 \pi}\left(\partial_{z^{r_{j}}}^{2} \sigma-\frac{1}{2}\left(\partial_{z^{r} j} \sigma\right)^{2}\right)\left(z_{j}, \bar{z}_{j}\right)\right)\right\rangle_{S^{1} \times S^{1}, h_{0}}\right. \\
& =\left\langle\prod_{j}\left(f^{\prime}\left(x_{j}\right)^{2} T_{r_{j}}\left(z_{j}, \bar{z}_{j}\right)+\frac{c}{48 \pi}\left(\frac{f^{\prime \prime \prime}}{f^{\prime}}-\left(\frac{f^{\prime \prime}}{f^{\prime}}\right)^{2}\right)\left(x_{j}\right)\right)\right\rangle_{S^{1} \times S^{1}, h_{0}}
\end{aligned}
$$

and the right-hand side does not represent correctly the Schwarzian-derivative terms of the Wick-rotated (5.2). In the Euclidian domain, the Schwarzian derivative appears in the transformation law of the energy-momentum components when one deals with holomorphic transformations $z \mapsto f(z)[40,57]$, but this is not the case here. A closer examination shows that

$$
\left\langle\prod_{j} T_{r_{j}}\left(x_{j} ;-i \tau_{j}\right)\right\rangle_{\mathrm{neq}}=\left\langle\prod_{j}\left(-T_{r_{j}}\left(z_{j}, \bar{z}_{j}\right)-\frac{c}{48 \pi} \mathcal{R}\left(z_{j}, \bar{z}_{j}\right)\right)\right\rangle_{S^{1} \times S^{1}, e^{\sigma} h_{0}},
$$

where $\mathcal{R}(z, \bar{z})=-\beta^{\prime \prime}(x) / \beta(x)$ is the scalar curvature of the metric $e^{\sigma} h_{0}$. This substantiates the comment made above about the need of caution.

\section{Conclusions}

We elaborated on the formula of [33] giving the full time evolution of the energy density and heat current from a nonequilibrium state with a preimposed temperature profile in the Luttinger model with local interactions. The formula was obtained in [33] by expanding the nonequilibrium state around the equilibrium to all orders. More details on the perturbative computation involving the exact calculation of complicated integrals, that may be interesting in its own right, were given. The main part of the paper was devoted to showing how the formula of [33], a result of the resummation of the perturbative series, may be obtained using Minkowskian conformal symmetries of the local Luttinger model. The idea was to use conformal transformations to map spatially inhomogeneous situations to homogeneous ones, straightening out a nonuniform temperature profile to a constant one. This led to a direct relation between nonequilibrium and equilibrium states, yielding the remarkable formula of [33] as a corollary. The CFT argument holds for a general class of unitary CFTs and could be applied to a wider class of nonequilibrium states that are preserved by the Schrödingerpicture evolution. The states in this class may be viewed as particular examples of simple generalized Gibbs states with local profiles, and they tend to ordinary generalized Gibbs states at long times, somewhat similarly as in the scenario recently advocated for integrable models where the evolution at certain length and time scales could be described by generalized hydrodynamics [14-18,20-23]. We obtained similar results also for CFTs with a U(1) 
current algebra (including the local Luttinger model itself) where we treated nonequilibrium states with temperature and chemical-potential profiles. Moreover, our results permit a more detailed analysis within CFT, compared to using the partitioning protocol studied before [27], of how a system starting in a state that looks like two different equilibria joint together evolves in time towards a nonequilibrium steady state described by a generalized Gibbs state.

As was discussed in Sect. 5, at least some families of the CFT nonequilibrium states that we studied in the present paper could be interpreted as providing a mesoscopic-scale description of dense nonrelativistic $1 d$ matter in macroscopic traps [29-32]. The dynamical correlators in such CFT states should similarly describe the corresponding nonrelativistic correlators at mesoscopic time scales both for after-quench and for equilibrium dynamics. The other way of arriving at the family of nonequilibrium CFT states that we considered is by reversing the logic of this paper. In the periodic-volume Minkowski CFT, the conformal symmetries (together with the gauge symmetries if a U(1) current algebra is present) are broken in the usual equilibria that are not preserved by the symmetries. Instead, the application of the symmetry transformations to the equilibrium states generates the family of nonequilibrium states that were studied here.

In the infinite volume, the states with kink-like profiles give access to the full counting statistics of the energy or charge transfers through the kinks, similarly to the states arising in the partitioning protocol [27]. Although such statistics in both approaches differ at finite times, they have the same long-time large deviations. This will be discussed elsewhere as it requires using different boundary conditions for finite volumes that allow one to avoid the duplication of kinks in the profiles. Finally, another interesting exercise, which was abundantly discussed in the similar context of quantum quenches [25], concerns the evolution of the entanglement entropy or negativity starting from states with profiles of the type consider here. By the replica trick, the latter may be extracted from nonequilibrium correlators of the twist primary fields in the replicated theory, to which our approach gives direct access. The analysis of the corresponding formulas is left for future research.

Acknowledgements All the authors profited from valuable input and encouragement by Joel Lebowitz and Vieri Mastropietro. They gratefully acknowledge that this paper would not have seen the light of day without them. They would also like to thank Jouko Mickelsson and Herbert Spohn for helpful discussions. E.L. acknowledges support by VR Grant No. 2016-05167. P.M. is thankful for financial support from P. F. Lindström's foundation (KTH travel scholarship VT-2017-0011 no. 4). The final version of the article has profited from pertinent comments of the anonymous referees who, in particular, drew our attention to the articles [29-32].

Open Access This article is distributed under the terms of the Creative Commons Attribution 4.0 International License (http://creativecommons.org/licenses/by/4.0/), which permits unrestricted use, distribution, and reproduction in any medium, provided you give appropriate credit to the original author(s) and the source, provide a link to the Creative Commons license, and indicate if changes were made.

\section{Appendix}

In this appendix we give a proof of Lemma 2.1.

We find it convenient to write the integrals in (2.7) as

$$
I_{n}\left(q_{1}, \ldots, q_{n}\right)=\frac{2}{v \bar{\beta}} \int_{\mathbb{R}} \sum_{\nu \in(2 \pi / \bar{\beta}) \mathbb{Z}} \prod_{j=0}^{n} f_{v}\left(p+Q_{j}\right) d p, \quad f_{v}(p)=\frac{v p}{\mathrm{i} v-v p}
$$


To compute these integrals we insert the Taylor series

$$
f_{v}\left(p+Q_{j}\right)=f_{v}(p)+f^{(1)}(p) Q_{j}+\frac{1}{2} f^{(2)}(p) Q_{j}^{2}+\ldots, \quad f_{v}^{(j \geq 1)}(p)=\frac{j ! v^{j} \mathrm{i} v}{(\mathrm{i} v-v p)^{j+1}}
$$

into the integrand and obtain

$$
I_{n}\left(q_{1}, \ldots, q_{n}\right)=I_{n}^{(n+1)}+I_{n}^{(n, 1)} \sum_{j=0}^{n} Q_{j}+\frac{1}{2} I_{n}^{(n, 0,1)} \sum_{j=0}^{n} Q_{j}^{2}+I_{n}^{(n, 2)} \sum_{0 \leq j<k \leq n} Q_{j} Q_{k}+R_{n}
$$

with the integrals

$$
I_{n}^{\left(m_{0}, m_{1}, \ldots, m_{k}\right)}=\frac{2}{v \bar{\beta}} \int_{\mathbb{R}} \sum_{\nu \in(2 \pi / \bar{\beta}) \mathbb{Z}} f_{v}(p)^{m_{0}} f_{\nu}^{(1)}(p)^{m_{1}} \ldots f_{v}^{(k)}(p)^{m_{k}} d p
$$

where $m_{j}=1,2, \ldots$ for $j=0,1, \ldots, k$ such that $\sum_{j} m_{j}=n+1$, and $R_{n}$ a linear combination of terms

$$
I_{n}^{\left(m_{0}, m_{1}, \ldots, m_{k}\right)} \prod_{j=1}^{k} Q_{\ell_{j, 1}}^{j} \ldots Q_{\ell_{j, m_{j}}}^{j}
$$

with indices $1 \leq \ell_{j, 1}<\cdots<\ell_{j, m_{j}} \leq n$ for $j=1,2, \ldots, k$ and $\left\{m_{j}\right\}$ such that $\sum_{j} j m_{j} \geq 3$.

To compute the integrals in (A.4) we define $M=\sum_{j} j m_{j}$ and insert the derivatives of $f_{v}(p)$ from (A.2). This gives

$$
\begin{aligned}
& I_{n}^{\left(m_{0}, m_{1}, \ldots, m_{k}\right)} \\
& =2 ! \cdots k ! \int_{\mathbb{R}} 2 v^{M+m_{0}-1} \frac{1}{\bar{\beta}} \sum_{v \in(2 \pi / \bar{\beta}) \mathbb{Z}} \frac{p^{m_{0}}(\mathrm{i} v)^{n-m_{0}+1}}{(\mathrm{i} v-v p)^{n+M+1}} d p \\
& =2 ! \ldots k ! \int_{\mathbb{R}} \sum_{\ell}^{n-m_{0}+1}\left(\begin{array}{c}
n-m_{0}+1 \\
\ell
\end{array}\right) 2 v^{M+m_{0}+\ell-1} \frac{1}{\bar{\beta}} \sum_{v \in(2 \pi / \bar{\beta}) \mathbb{Z}} \frac{p^{m_{0}+\ell}}{(\mathrm{i} v-v p)^{M+m_{0}+\ell}} d p \\
& =2 ! \ldots k ! \int_{\mathbb{R}} \sum_{\ell=0}^{n-m_{0}+1}\left(\begin{array}{c}
n-m_{0}+1 \\
\ell
\end{array}\right) \frac{(v \bar{\beta})^{M-2} s^{m_{0}+\ell}}{\left(M+m_{0}+\ell-1\right) !} \frac{d^{M+m_{0}+\ell-1}}{d s^{M+m_{0}+\ell-1}}\left(\operatorname{coth}\left(\frac{1}{2} s\right)\right) d s .
\end{aligned}
$$

In the second equality we wrote $(\mathrm{i} v)^{n-m_{0}+1}=(\mathrm{i} v-v p+v p)^{n-m_{0}+1}$ to expand into a binomial series, and in the third we summed the bosonic Matsubara frequencies $v$ using the Mittag-Leffler series of $\operatorname{coth}(v \bar{\beta} p / 2)$ and changed variables to $s=v \bar{\beta} p$. We note that, for $M \geq 1$, the integrand of the last integral is singular, but the singularity is removable, i.e., one can replace $\operatorname{coth}(s / 2)$ by $\operatorname{coth}(s / 2)-2 / s$ without changing the result. To further compute these integrals we use partial integrations. We find that the integrals in (A.6) are zero for $M \geq 3$, which implies $R_{n}=0$. The remaining integrals $I_{n}^{(n+1)}, I_{n}^{(n, 1)}, I_{n}^{(n, 0,1)}$, and $I_{n}^{(n-1,2)}$ are found by straightforward computations. Inserting them into (A.3) we obtain the result in Lemma 2.1. 


\section{References}

1. Rieder, Z., Lebowitz, J.L., Lieb, E.: Properties of a harmonic crystal in a stationary nonequilibrium state. J. Math. Phys. 8, 1073 (1967)

2. Spohn, H., Lebowitz, J.L.: Stationary non-equilibrium states of infinite harmonic systems. Commun. Math. Phys. 54, 97 (1977)

3. Zotos, X., Naef, F., Prelovsek, P.: Transport and conservation laws. Phys. Rev. B 55, 11029 (1997)

4. Ho, T.G., Araki, H.: Asymptotic time evolution of a partitioned infinite two-sided isotropic $X Y$-chain. Tr. Mat. Inst. Steklova 228, 203 (2000)

5. Ogata, Y.: Nonequilibrium properties in the transverse $X X$ chain. Phys. Rev. E 66, 016135 (2002)

6. Aschbacher, W.H., Pillet, C.-A.: Non-equilibrium steady states of the $X Y$ chain. J. Stat. Phys. 112, 1153 (2003)

7. Giamarchi, T.: Quantum Physics in One Dimension. Oxford University Press, Oxford (2004)

8. Zotos, X.: Issues on the transport of one dimensional systems. J. Phys. Soc. Jpn. Suppl. 74, 173 (2005)

9. Sirker, J., Pereira, R.G., Affleck, I.: Diffusion and ballistic transport in one-dimensional quantum systems. Phys. Rev. Lett. 103, 216602 (2009)

10. Sirker, J., Pereira, R.G., Affleck, I.: Conservation laws, integrability, and transport in one-dimensional quantum systems. Phys. Rev. B 83, 035115 (2011)

11. Bloch, I., Dalibard, J., Zwerger, W.: Many-body physics with ultracold gases. Rev. Mod. Phys. 80, 885 (2008)

12. Polkovnikov, A., Sengupta, K., Silva, A., Vengalattore, M.: Colloquium: nonequilibrium dynamics of closed interacting quantum systems. Rev. Mod. Phys. 83, 863 (2011)

13. Cazalilla, M.A., Chung, M.-C.: Quantum quenches in the Luttinger model and its close relatives. J. Stat. Mech. 064004 (2016)

14. Bertini, B., Collura, M., De Nardis, J., Fagotti, M.: Transport in out-of-equilibrium $X X Z$ chains: exact profiles of charges and currents. Phys. Rev. Lett. 117, 207201 (2016)

15. Castro-Alvaredo, O.A., Doyon, B., Yoshimura, T.: Emergent hydrodynamics in integrable quantum systems out of equilibrium. Phys. Rev. X 6, 041065 (2016)

16. Bulchandani, V.B., Vasseur, R., Karrasch, C., Moore, J.E.: Bethe-Boltzmann hydrodynamics and spin transport in the XXZ chain. arXiv:1702.06146 [cond-mat.stat-mech] (2017)

17. Doyon, B., Spohn, H., Yoshimura, T.: A geometric viewpoint on generalized hydrodynamics. arXiv:1704.04409 [cond-mat.stat-mech] (2017)

18. Doyon, B., Spohn, H.: Drude weight for the Lieb-Liniger Bose gas. arXiv:1705.08141 [cond-mat.statmech] (2017)

19. Spohn, H.: Interacting and noninteracting integrable systems. arXiv:1707.02159 [cond-mat.stat-mech] (2017)

20. Doyon, B., Yoshimura, T.: A note on generalized hydrodynamics: inhomogeneous fields and other concepts. SciPost Phys. 2, 014 (2017)

21. Ilievski, E., De Nardis, J.: Ballistic transport in the one-dimensional Hubbard model: the hydrodynamic approach. Phys. Rev. B 96, 081118(R) (2017)

22. Caux, J.-S., Doyon, B., Dubail, J., Konik, R., Yoshimura, T.: Hydrodynamics of the interacting Bose gas in the Quantum Newton Cradle setup. arXiv:1711.00873 [cond-mat.stat-mech] (2017)

23. Doyon, B.: Exact large-scale correlations in integrable systems out of equilibrium. arXiv:1711.04568 [math-ph] (2017)

24. Calabrese, P., Cardy, J.: Time dependence of correlation functions following a quantum quench. Phys. Rev. Lett. 96, 136801 (2006)

25. Calabrese, P., Cardy, J.: Quantum quenches in 1+1 dimensional conformal field theories. J. Stat. Mech. 064003 (2016)

26. Bernard, D., Doyon, B.: Energy flow in non-equilibrium conformal field theory. J. Phys. A 45, 362001 (2012)

27. Bernard, D., Doyon, B.: Conformal field theory out of equilibrium: a review. J. Stat. Mech. 064005 (2016)

28. Hollands, S., Longo, R.: Non-equilibrium thermodynamics and conformal field theory. Commun. Math. Phys. 357, 43 (2018)

29. Dubail, J., Stéphan, J.-M., Viti, J., Calabrese, P.: Conformal field theory for inhomogeneous onedimensional quantum systems: the example of non-interacting Fermi gases. SciPost Phys. 2, 002 (2017)

30. Brun, Y., Dubail, J.: One-particle density matrix of trapped one-dimensional impenetrable bosons from conformal invariance. SciPost Phys. 2, 012 (2017)

31. Dubail, J., Stéphan, J.-M., Calabrese, P.: Emergence of curved light-cones in a class of inhomogeneous Luttinger liquids. SciPost Phys. 3, 019 (2017) 
32. Brun, Y., Dubail, J.: The Inhomogeneous Gaussian Free Field, with application to ground state correlations of trapped 1d Bose gases. arXiv:1712.05262 [cond-mat.stat-mech] (2017)

33. Langmann, E., Lebowitz, J.L., Mastropietro, V., Moosavi, P.: Time evolution of the Luttinger model with nonuniform temperature profile. Phys. Rev. B 95, 235142 (2017)

34. Sotiriadis, S., Cardy, J.: Inhomogeneous quantum quenches. J. Stat. Mech. P1, 2008 (1003)

35. Vidmar, L., Rigol, M.: Generalized Gibbs ensemble in integrable lattice models. J. Stat. Mech. 064007 (2016)

36. Langmann, E., Lebowitz, J.L., Mastropietro, V., Moosavi, P.: Steady states and universal conductance in a quenched Luttinger model. Commun. Math. Phys. 349, 551 (2017)

37. Mattis, D.C., Lieb, E.H.: Exact solution of a many-fermion system and its associated boson field. J. Math. Phys. 6, 304 (1965)

38. Langmann, E., Moosavi, P.: Construction by bosonization of a fermion-phonon model. J. Math. Phys. 56, 091902 (2015)

39. Voit, J.: One-dimensional Fermi liquids. Rep. Prog. Phys. 58, 977 (1995)

40. Di Francesco, P., Mathieu, P., Sénéchal, D.: Conformal Field Theory. Springer, Berlin (1997)

41. Callen, H.N.: Thermodynamics and an Introduction to Thermostatics. Wiley, New York (1985)

42. Bernard, D., Doyon, B.: Diffusion and signatures of localization in stochastic conformal field theory. Phys. Rev. Lett. 119, 110201 (2017)

43. Mastropietro, V., Wang, Z.: Quantum quench for inhomogeneous states in the nonlocal Luttinger model. Phys. Rev. B 91, 085123 (2015)

44. Ruijsenaars, S.N.M.: On Bogoliubov transformations for systems of relativistic charged particles. J. Math. Phys. 18, 517 (1977)

45. Ruijsenaars, S.N.M.: On Bogoliubov transformations. II. The general case. Ann. Phys. 116, 105 (1978)

46. Grosse, H., Langmann, E.: A superversion of quasifree second quantization. I. Charged particles. J. Math. Phys. 33, 1032 (1992)

47. Goodman, R., Wallach, N.R.: Projective unitary positive-energy representations of Diff $\left(S^{1}\right)$. J. Funct. Anal. 63, 299 (1985)

48. Vasseur, R., Karrasch, C., Moore, J.E.: Expansion potentials for exact far-from-equilibrium spreading of particles and energy. Phys. Rev. Lett. 115, 267201 (2015)

49. Bernard, D., Doyon, B.: A hydrodynamic approach to non-equilibrium conformal field theories. J. Stat. Mech. 033104 (2016)

50. Goodman, R., Wallach, N.R.: Structure and unitary cocycle representations of loop groups and the group of diffeomorphisms of the circle. J. Reine Angew. Math. 347, 69 (1984)

51. Maslov, D.L., Stone, M.: Landauer conductance of Luttinger liquids with leads. Phys. Rev. B 52, R5539 (1995)

52. Alekseev, A.Y., Cheianov, V.V., Fröhlich, J.: Comparing conductance quantization in quantum wires and quantum Hall systems. Phys. Rev. B 54, R17320 (1996)

53. Kawabata, A.: On the renormalization of conductance in Tomonaga-Luttinger liquid. J. Phys. Soc. Jpn. 65, 30 (1996)

54. Tarucha, S., Honda, T., Saku, T.: Reduction of quantized conductance at low temperatures observed in 2 to $10 \mu \mathrm{m}$-long quantum wires. Sol. State Commun. 94, 413 (1995)

55. Kane, C.L., Fisher, M.P.A.: Transport in a one-channel Luttinger liquid. Phys. Rev. Lett. 68, 1220 (1992)

56. Langmann, E., Moosavi, P.: in preparation

57. Gawȩdzki, K.: Lectures on conformal field theory. In: Deligne, P., et al. (eds.) Quantum Fields and Strings: A Course for Mathematicians, pp. 727-805. Providence, AMS-IAS (1999) 